\title{
The quadratic MITC plate and MITC shell elements in plate bending
}

\author{
Phill-Seung Lee ${ }^{\mathrm{a}}$, Klaus-Jürgen Bathe ${ }^{\mathrm{b}, *}$ \\ ${ }^{a}$ Division of Ocean Systems Engineering, Korea Advanced Institute of Science and Technology, Daejeon 305-701, Korea \\ ${ }^{\mathrm{b}}$ Department of Mechanical Engineering, Massachusetts Institute of Technology, Cambridge, MA 02139, USA
}

\section{A R T I C L E I N F O}

\section{Article history:}

Received 21 September 2009

Accepted 10 December 2009

Available online 6 January 2010

\section{Keywords:}

Plate

Shell

MITC

Mixed formulation

Finite element

Locking

Optimal convergence

\begin{abstract}
A B S T R A C T
The analysis of plates can be achieved using the quadratic MITC plate or MITC shell elements. The plate elements have a strong mathematical basis and have been shown to be optimal in their convergence behavior, theoretically and numerically. The shell elements have not (yet) been analyzed mathematically in depth for their rates of convergence, with the plate/shell thickness varying, but have been shown numerically to perform well. Since the shell elements are general and can be used for linear and nonlinear analyses of plates and shells, it is important to identify the differences in the performance of these elements when compared to the plate elements. We briefly review the quadratic quadrilateral and triangular MITC plate and shell elements and study their performances in linear plate analyses.
\end{abstract}

(c) 2009 Elsevier Ltd. All rights reserved.

\section{Introduction}

The analysis of plates and shells is ubiquitous and the development of effective finite elements has attracted a large amount of research, see for example Refs. [1-3]. While, practically, optimal plate finite elements are now available, the development of effective shell elements is a much more difficult task [3].

Numerous plate finite elements have been proposed. These can be used for small displacement analyses. However, in case the plate structure carries also membrane forces, it is most expedient to model the structure as a shell. Furthermore, when the response includes large displacements, a shell element to model the structure is best used since during the response the structural behavior changes from that of a plate to that of a shell.

Since a plate is, in fact, a simple flat shell, in practice, frequently shell elements are used to model the bending of plates, even for infinitesimally small displacement solutions. In this way, the same element is employed for all plate and shell analyses, which makes the modeling of such structures uniform and effective.

The first shell element developments were based on superimposing plate bending and membrane plane stress stiffness matrices $[1,2]$. While such elements can be effective for some applications, they need to be used with care since proper curved shell behavior is not represented and the discretization may not converge as the element size decreases, see Ref. [3] for an example.

\footnotetext{
* Corresponding author.

E-mail address: kjb@mit.edu (K.J. Bathe).
}

Some powerful shell elements for specific applications were developed using restricted shell theories, like assuming small Gaussian curvatures, and small displacements, but these discretization schemes are limited in that they can only be used for certain limited applications. Such elements are hardly used in practice.

General and effective shell elements are the mixed-interpolated tensorial components, or MITC, elements proposed in Refs. [4-9]. These elements are based on choosing appropriate strain interpolations for a given displacement interpolation and then tying the strain interpolations to the displacements at specific tying points, see also Refs. $[2,3,10,11]$. Hence the final degrees of freedom are as for the displacement-based elements. The theoretical basis of the MITC shell elements has been published [2-14] and the elements have been tested numerically in the solution of various well-chosen test problems [3-11,13-16]; for the problems used and convergence measures, see in particular $[3,16,17]$. However, a mathematical convergence analysis including the effect of the shell thickness has, so far, been out of reach. Indeed, deep mathematical analyses of shell elements have only been achieved when performed on simple specific shell geometries, boundary conditions, and loadings, see e.g. Refs. $[18,19]$. The reason is that a general analysis would have to consider any shell geometry, thickness, loading and boundary conditions. Such an all-encompassing mathematical analysis is extremely difficult to perform.

In this paper we focus on the MITC plate and MITC shell elements that are based on quadratic displacement interpolations. We do not consider the four-node bilinear MITC4 shell element because the same element is used for plates and shells [2]. The MITC plate elements, see Refs. [2,20-22], constructed in principle like 
the shell elements, have a strong mathematical basis for linear plate analyses and show optimal convergence behavior, independent of the plate thickness, in mathematical analyses and numerical tests [23-26]. The disadvantage of these elements is that they have internal nodes that only carry rotational degrees of freedom. The use of these internal nodal rotations renders the quadratic MITC plate elements difficult to extend to shell and large deformation analyses. Also, in practice, the same degrees of freedom are best used at each node of an element.

It should be realized that, intrinsically, the development of effective shell elements is much more difficult than the development of plate elements because of the curvature effects in a shell, and the sensitivity of shells to curvature, boundary and loading conditions $[3,15]$. Of course, once a shell element has been developed, the element is usually also tested in plate solutions. Considering the use of the MITC shell elements and the fact that the MITC plate elements have an optimal convergence behavior, a natural question to ask is therefore "How much more powerful are the MITC plate bending elements when compared to the MITC shell elements in the linear analysis of plates?"

The objective in this paper is to address this question by comparing numerically the performance of the quadratic MITC plate and MITC shell elements in the linear analysis of plates. In the next sections we first briefly review the plate and shell elements, and we then study the relative performance of the elements in some typical plate analyses. We focus on the comparison of the triangular MITC7 plate and MITC6 shell elements, and the quadrilateral MITC9 plate and MITC9 shell elements in numerical tests. The elements of even higher order are hardly used in engineering practice
[27]. A mathematical convergence analysis of the MITC shell elements when used in linear plate analyses could be the objective of another paper.

\section{The MITC plate and MITC shell elements}

As well known, displacement-based shell elements using the Reissner/Mindlin kinematical assumption and the plane stress assumption for the stress state are not effective because they lock in shear and membrane behavior [2]. The basic idea in the formulation of the MITC elements is to use the usual displacement interpolations for plates and shells, as for displacement-based elements, and 'judiciously' choose a corresponding strain interpolation. The unknown parameters attached to the strain interpolations are then tied to the strain components obtained from the displacement interpolations at 'judiciously' chosen tying points. In this way, the strain parameters are eliminated and the only degrees of freedom of the element are the usual displacement and rotation degrees of freedom at the nodes, as for the displacement-based elements [2].

This process of formulating elements can be used when employing Reissner/Mindlin plate theory to reach plate elements, and when using 'the basic shell model' underlying general shell elements $[3,11,12]$ to reach shell elements. In the first case, the MITC plate elements and in the second case the MITC shell elements are obtained.

The key to success of an MITC element formulation lies in the 'judicious' choices for the strain interpolations and the tying points for a given displacement interpolation. In essence, these choices

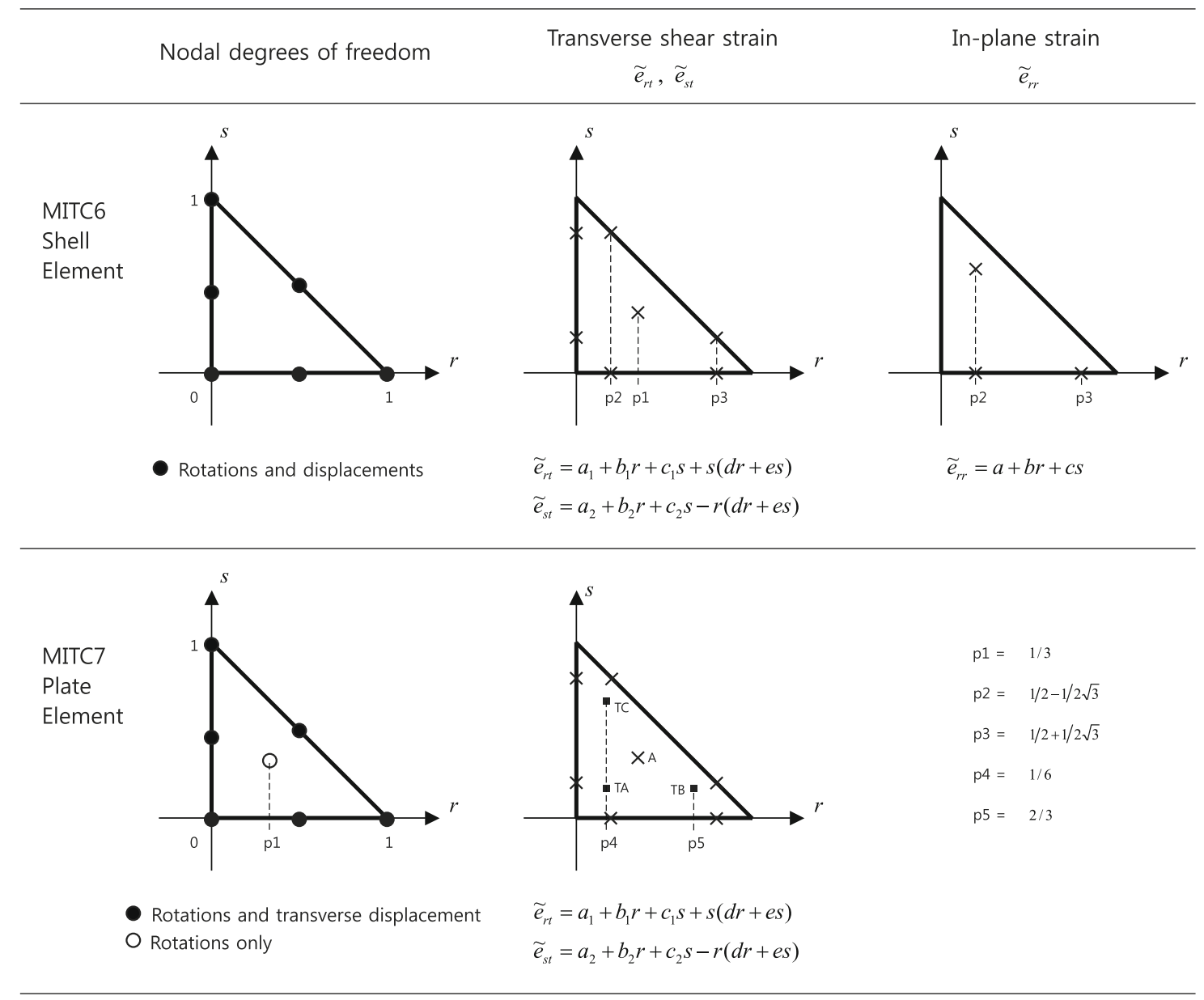

Fig. 1. Nodal degrees of freedom, interpolation functions for the covariant strain components, and the tying points for the triangular elements considered. 
shall extract from the displacement-based strains, that make the element almost useless, those components that render the element to be effective: for plate elements, the aim is to remove shear locking, and for shell elements, the aim is to remove shear and membrane locking, where membrane locking in shell analysis is usually a more severe problem. At the same time, consistency in all strain terms needs to be preserved.

It should be realized that the MITC formulation is quite different from the 'enhanced assumed strain' formulation or 'method of incompatible modes' [28]. Both techniques start with the displacement formulation and aim to improve its predictive capability. But, whereas in the MITC formulation, the strain assumptions inherently used in the displacement formulation are improved by not including certain terms of the displacement-based strain space, in the enhanced assumed strain formulations, new strain fields are added to those already inherently used in the displacement formulation. If this addition of strain terms actually results in a subtraction, in some cases, the same element matrices may be reached. However, more generally, the resulting elements are different and considerably more computations are required in the enhanced assumed strain formulations. Furthermore, an enhanced assumed strain formulation may be unstable in large deformation analysis when the same formulation is stable in linear analysis, see for example Ref. [29].

The formulation of the MITC elements corresponds in fact to an application of the $\mathrm{Hu}-$ Washizu variational principle $[2,4,13,14]$. However, this fact in itself, of course, does not mean that a formulated element is effective. The efficiency of an element needs to be analyzed mathematically, as far as possible, and tested numerically.

As mentioned already, the elements we consider here have been published before. Hence, in the following sections we only focus on the specific items of the element formulations that are needed for the understanding of our numerical results.

\subsection{Interpolation schemes for MITC plate and shell finite elements}

Figs. 1 and 2 show the nodes, nodal degrees of freedom, and summarize the interpolations used for the MITC plate and MITC shell elements that we test numerically in this paper. We note that the MITC7 and MITC9 plate elements have an internal node with only rotational degrees of freedom, whereas the MITC6 and MITC9

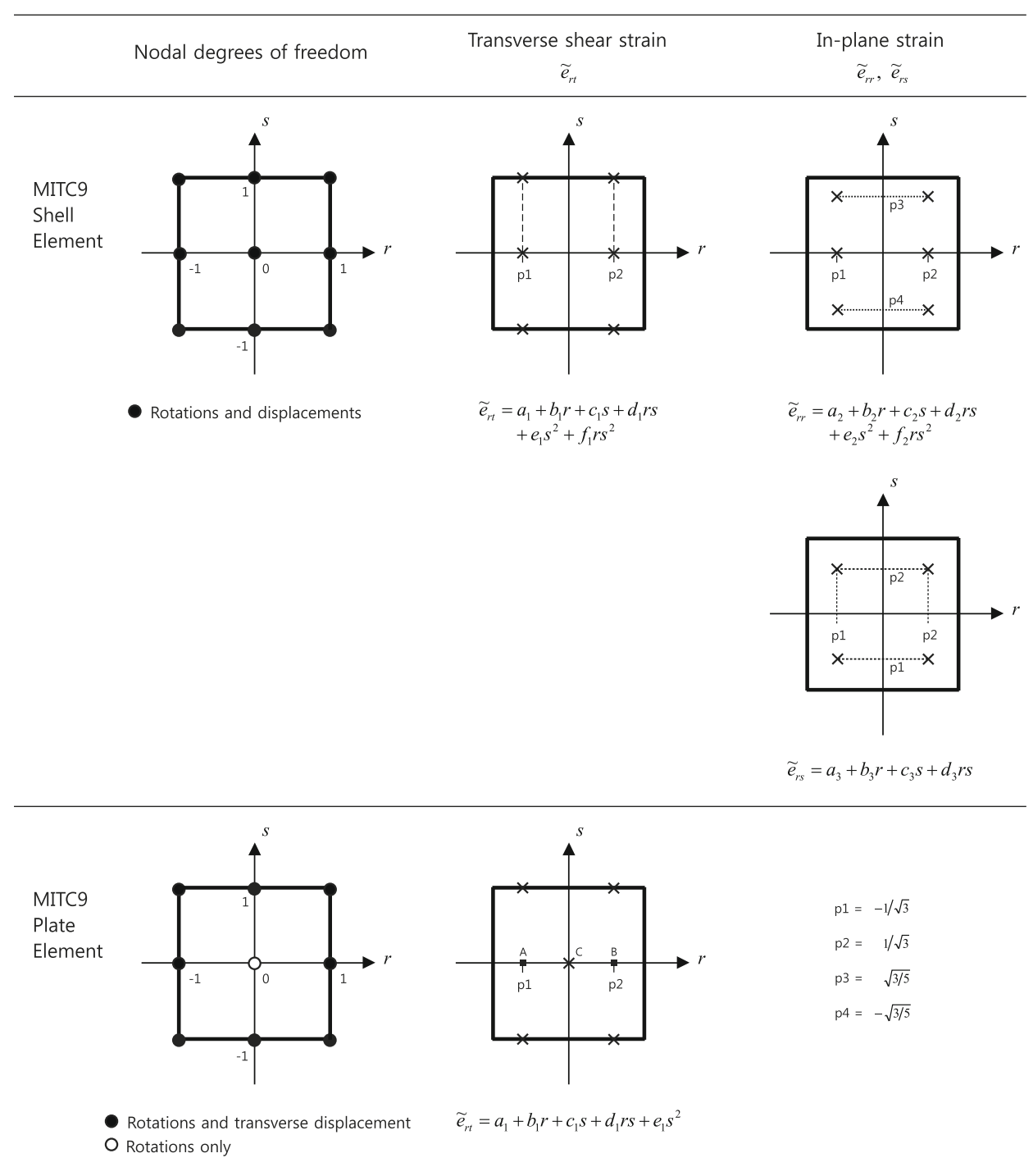

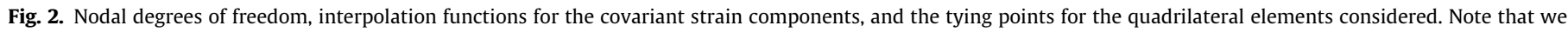
use the improved MITC9 shell element and not the original MITC9 shell element [7]. 
shell elements carry the same degrees of freedom at each node: for plate analysis, the transverse displacement and two section rotations. The MITC plate and shell elements are therefore clearly different. Note that in-plane strains are assumed in the MITC shell elements to avoid membrane locking, but such scheme is not used (and not needed) for the MITC plate elements.

The MITC7 plate and MITC6 shell elements use the same interpolation polynomials for the transverse shear strain field. However, the coefficients in the interpolation functions are different because, for the MITC7 plate element, the coefficient for the interpolation of the transverse shear strain using the element center $(r=s=1 / 3)$ is evaluated by

$$
\begin{aligned}
& \left.\tilde{e}_{r t}\right|_{A}=\left(\left.e_{r t}\right|_{T A}+\left.e_{r t}\right|_{T B}+\left.e_{r t}\right|_{T C}\right) / 3, \\
& \left.\tilde{e}_{s t}\right|_{A}=\left(\left.e_{s t}\right|_{T A}+\left.e_{s t}\right|_{T B}+\left.e_{s t}\right|_{T C}\right) / 3,
\end{aligned}
$$

where the over-curl denotes the interpolation value used, and the displacement-based strains do not carry the curl.

As shown in Fig. 2, the MITC9 plate and MITC9 shell finite elements have different interpolation functions and tying points for the transverse shear strains. For the MITC9 plate element, the transverse shear strain $\tilde{e}_{r t}$ at the element center is evaluated by

$\left.\tilde{e}_{r t}\right|_{C}=\left(\left.e_{r t}\right|_{A}+\left.e_{r t}\right|_{B}\right) / 2$.
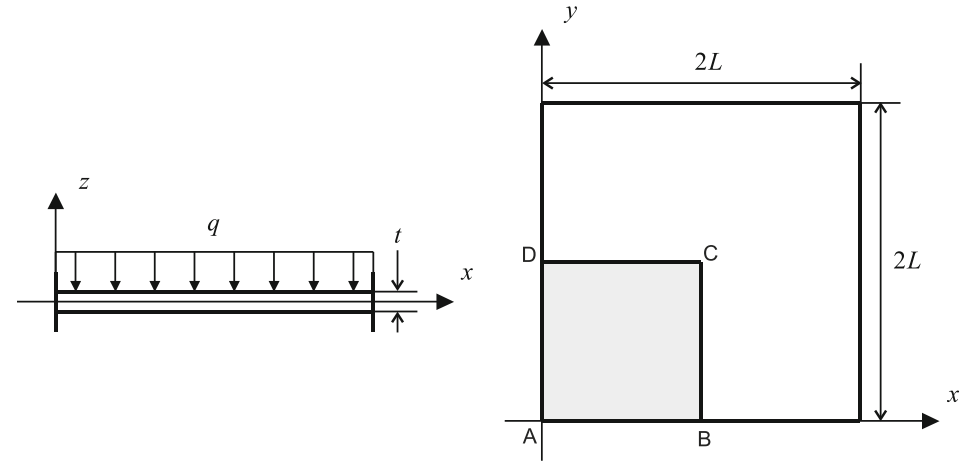

(a)

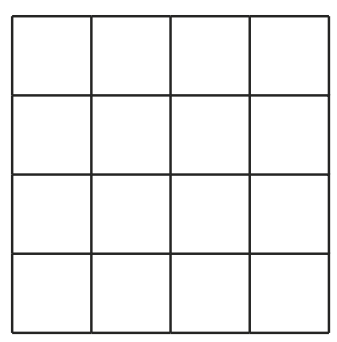

(b)

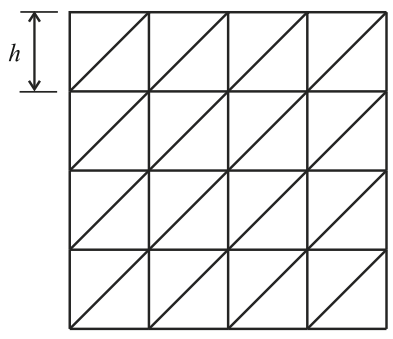

(c)

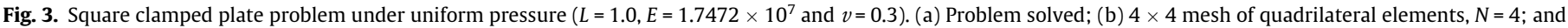
(c) $4 \times 4$ mesh of triangular elements, $N=4$.
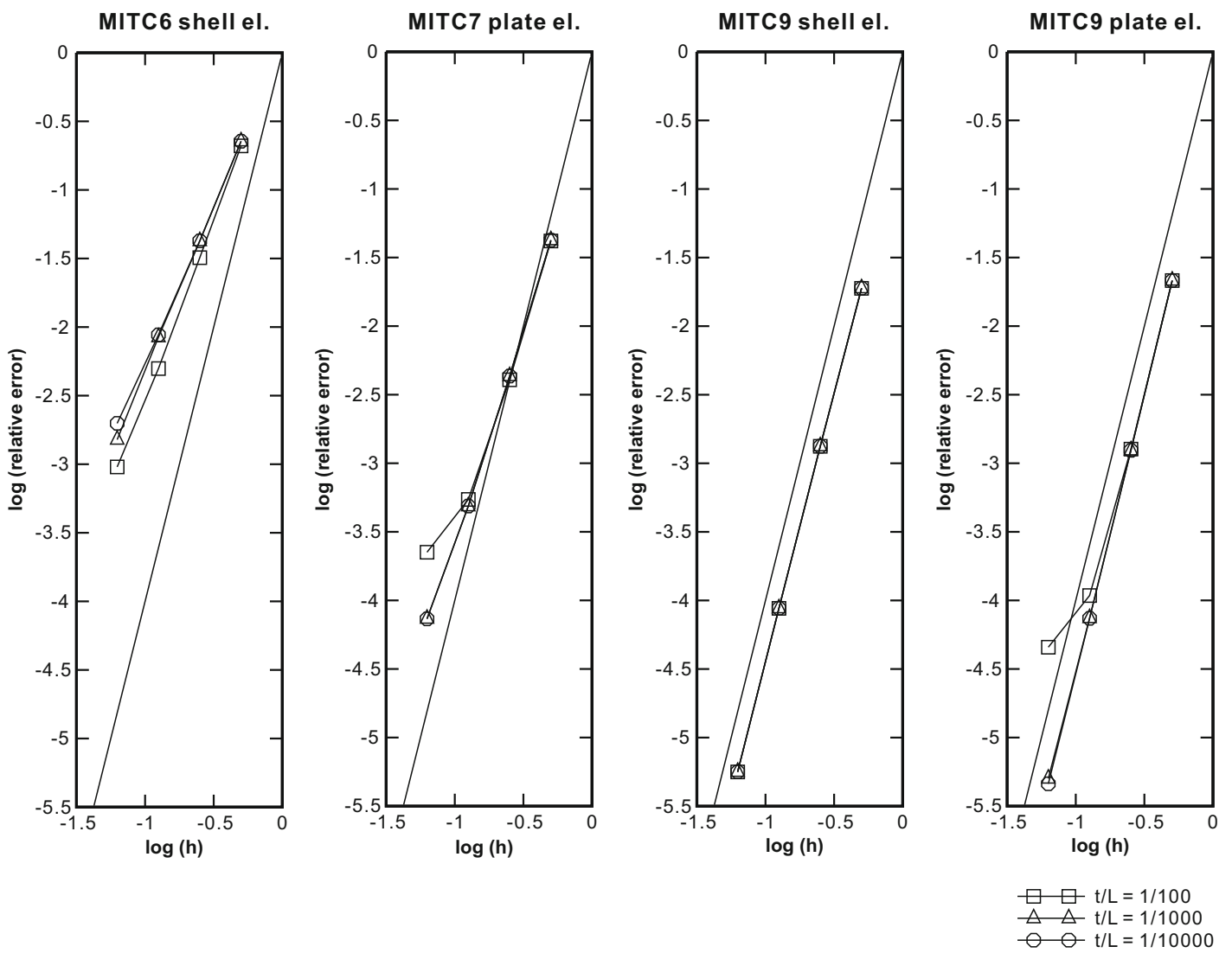

Fig. 4. Convergence curves for the square clamped plate problem. The s-norm is used. The solid lines represent the theoretical convergence in Eq. (5). 

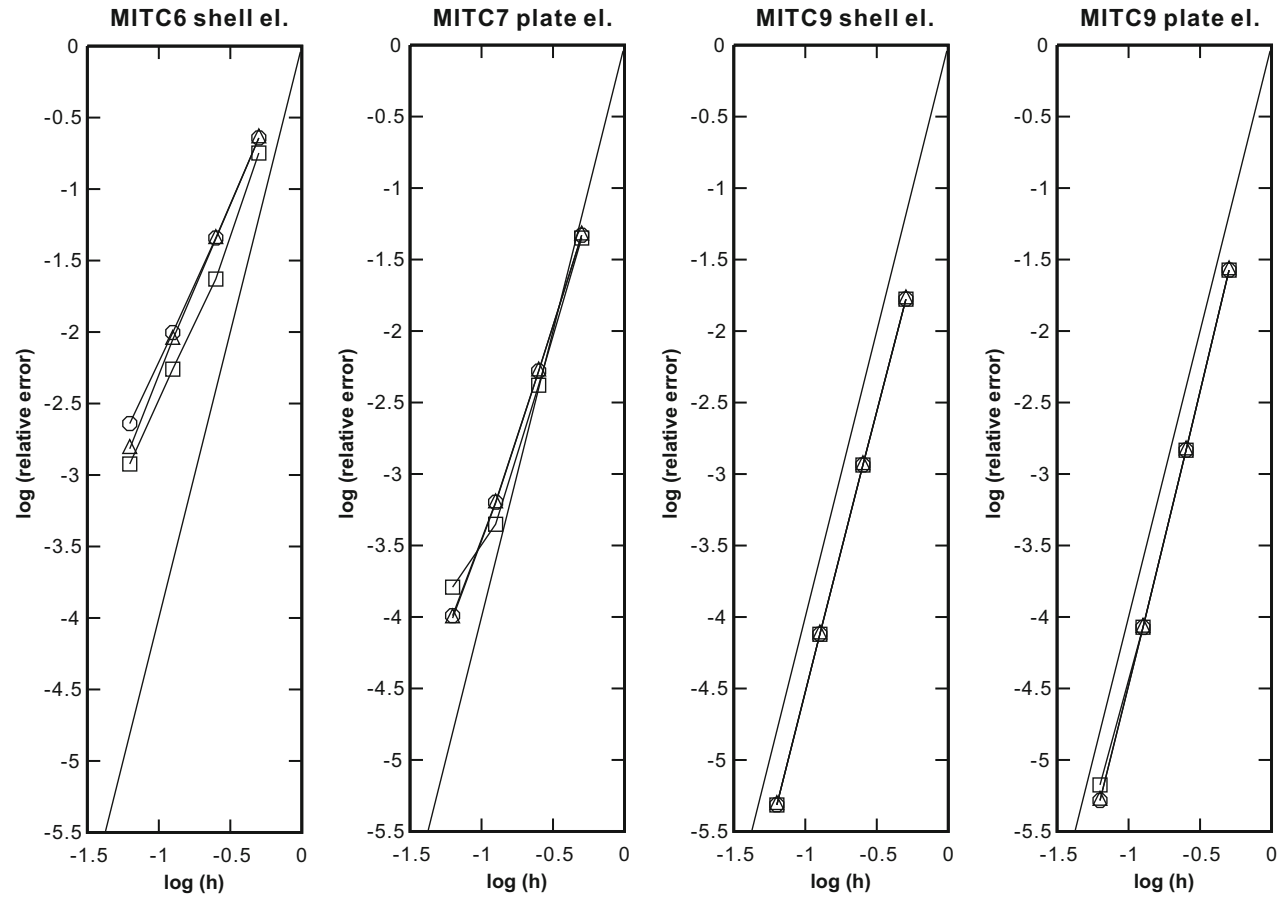

$\square \mathrm{t} / \mathrm{L}=1 / 100$
$\triangle \triangle \mathrm{L} / \mathrm{L}=1 / 1000$
$\Delta \triangle \mathrm{O} / \mathrm{L}=1 / 10000$

Fig. 5. Convergence of rotations for the square clamped plate problem. The solid lines represent the theoretical convergence in Eq. (3).
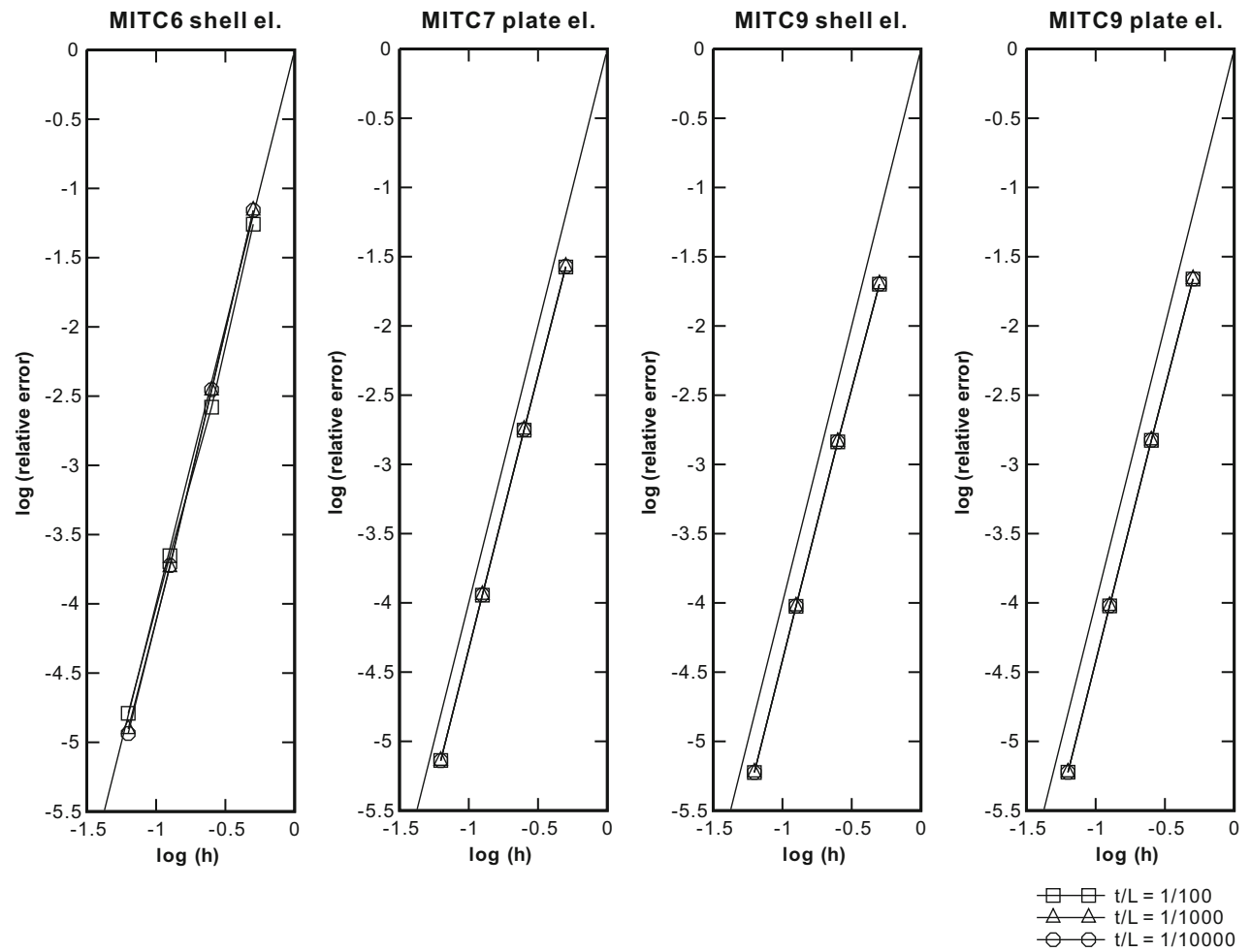

Fig. 6. Convergence of gradient of transverse displacement for the square clamped plate problem. The solid lines represent the theoretical convergence in Eq. (3).

Considering these figures, the not-shown strain components are interpolated in a symmetric manner.

We should note that for the MITC7 and MITC9 plate element formulations, instead of the integral tying over the element surfaces prescribed theoretically for the MITC7 and MITC9 plate element formulations, we use Eqs. (1) and (2), which was used and shown to be effective in Ref. [21].

We also note that, in this study, the shear correction factor $k$ with value $k=1$ is used for all MITC plate and MITC shell finite element solutions [2]. 

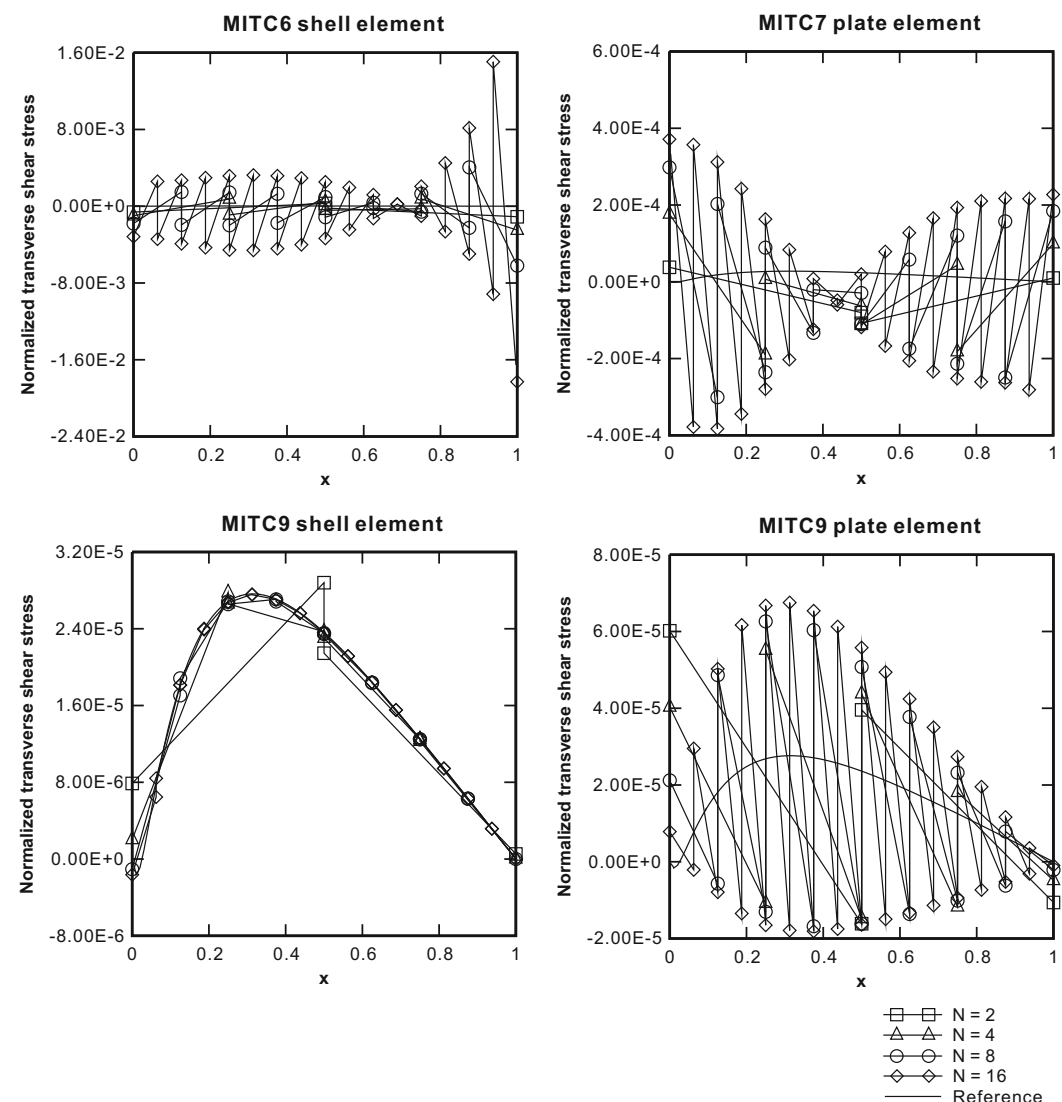

Fig. 7. Distribution of the normalized transverse shear stress- $x z$ along $A B(t / L=1 / 10,000)$.
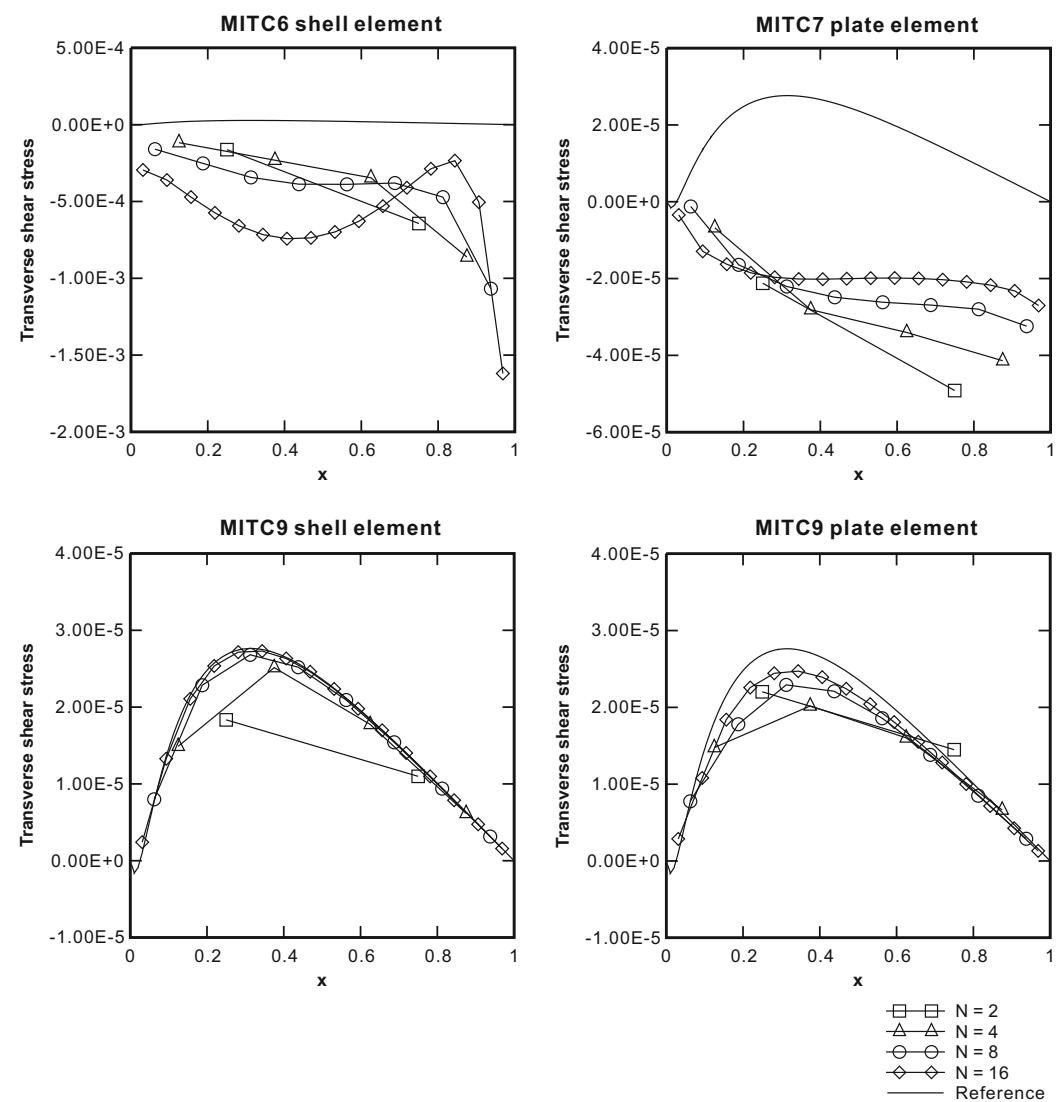

Fig. 8. Distribution of the normalized transverse shear stress- $x z$ along $A B(t / L=1 / 10,000)$. The stress is sampled at the mid-points of the element edges. 

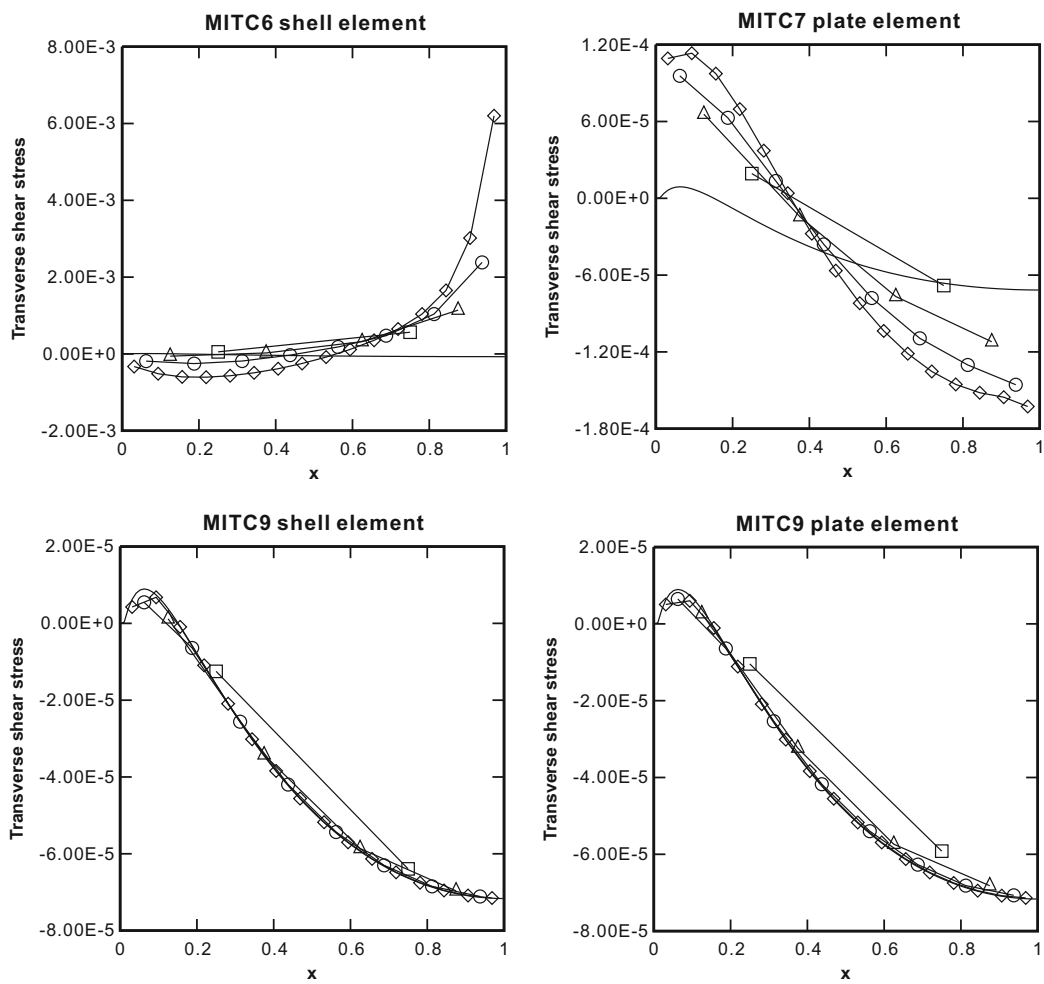

마 $N=2$

$\bigcirc \bigcirc N=8$

Reference

Fig. 9. Distribution of the normalized transverse shear stress- $y z$ along $A B(t / L=1 / 10,000)$. The stress is sampled at the mid-points of the element edges.
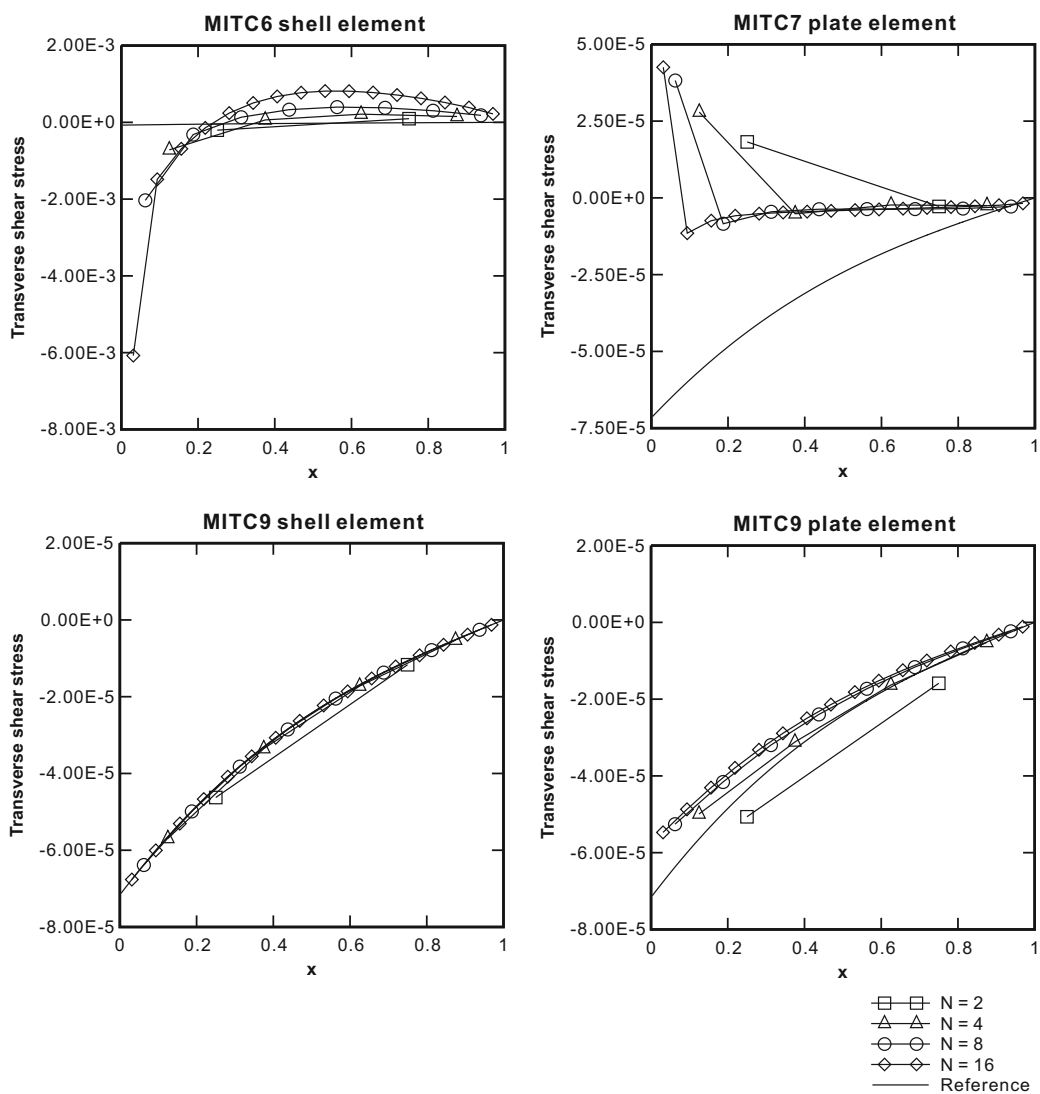

Fig. 10. Distribution of the normalized transverse shear stress- $x z$ along $D C(t / L=1 / 10,000)$. The stress is sampled at the mid-points of the element edges. 

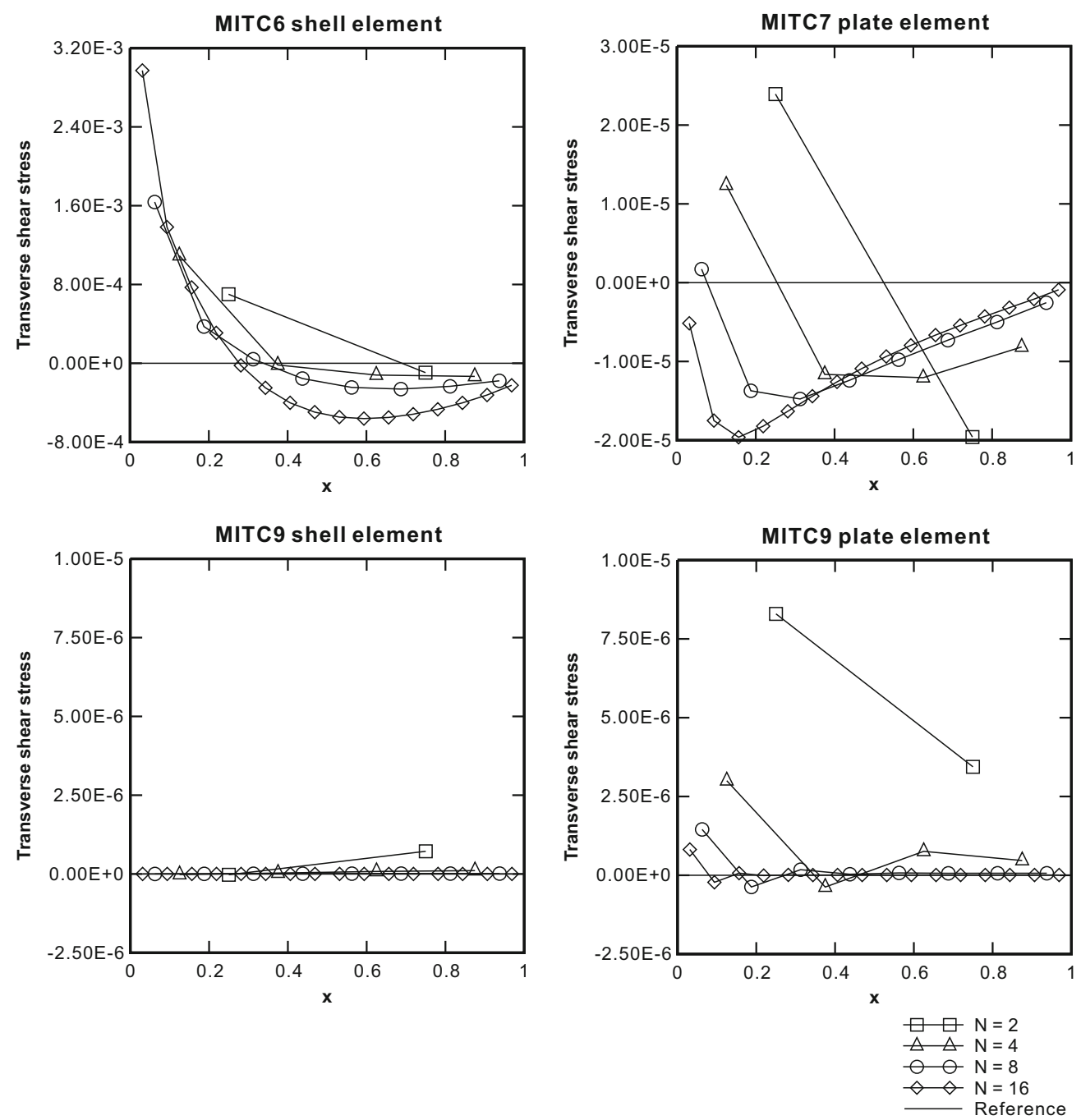

Fig. 11. Distribution of the normalized transverse shear stress-yz along DC $(t / L=1 / 10,000)$. The stress is sampled at the mid-points of the element edges.

\subsection{Convergence estimates}

The theoretical convergence behavior of the MITC plate and MITC shell elements ideally reached in practice by the elements considered in this study (but not yet proven to hold for the MITC shell elements), is given by

$$
\begin{aligned}
& \left\|\vec{\theta}-\vec{\theta}_{h}\right\|_{1}^{2} \cong c h^{4}, \\
& \left\|\vec{\nabla} w-\vec{\nabla} w_{h}\right\|_{0}^{2} \cong c h^{4},
\end{aligned}
$$

where $\vec{\theta}$ and $\vec{\nabla} w$ are the rotation vector and the gradient vector of the transverse displacement of the exact solution

$$
\vec{\theta}=\left[\begin{array}{l}
\theta_{x} \\
\theta_{y}
\end{array}\right] \quad \text { and } \quad \vec{\nabla} w=\left[\begin{array}{l}
w_{, x} \\
w_{, y}
\end{array}\right],
$$

and $\vec{\theta}_{h}$ and $\vec{\nabla} w_{h}$ are the vectors of the finite element solution. In Eq. (3), $\|\cdot\|_{1}$ and $\|\cdot\|_{0}$ denote the Sobolev norms [2,3], $h$ is the element size and $c$ is a constant, different in each equation.

We will also use the $s$-norm $\left(\|\cdot\|_{s}\right)$ proposed by Hiller and Bathe to measure convergence of mixed formulations [7-9,16,17].

$\left\|\vec{u}-\vec{u}_{h}\right\|_{s}^{2} \cong c h^{4}$ where $\vec{u}$ denotes the exact solution and $\vec{u}_{h}$ denotes the finite element solution.

We will evaluate our numerical solutions with these three convergence rate estimates. To measure the convergence of the finite elements in the plate bending problems with various plate thicknesses, we study the relative errors defined as

$$
\begin{aligned}
E_{\vec{\theta}} & =\frac{\left\|\vec{\theta}_{r e f}-\vec{\theta}_{h}\right\|_{1}^{2}}{\left\|\vec{\theta}_{r e f}\right\|_{1}^{2}}, \quad E_{\vec{\nabla} w}=\frac{\left\|\vec{\nabla} w_{r e f}-\vec{\nabla} w_{h}\right\|_{0}^{2}}{\left\|\vec{\nabla} w_{r e f}\right\|_{0}^{2}} \text { and } E_{s} \\
& =\frac{\left\|\vec{u}_{r e f}-\vec{u}_{h}\right\|_{s}^{2}}{\left\|\vec{u}_{r e f}\right\|_{s}^{2}} .
\end{aligned}
$$

To calculate the quantities in Eq. (6), the finite element solutions $\left(\vec{\theta}_{\text {ref }}, \vec{\nabla} w_{\text {ref }}, \vec{u}_{\text {ref }}\right)$ using very fine reference meshes are employed instead of the exact solutions. The calculation procedure to evaluate the error measures using the reference and target meshes is presented in Ref. [17].

In addition to giving the above convergence curves, we also present results in shear stress predictions. As well known, the shear stresses are very difficult to accurately calculate for thin plates and for the 'continuous' problem theoretically do not even converge in the $L^{2}$ norm as $t / L \rightarrow 0$ [3]. Hence we simply plot the results along certain sections of the plates to show visually the convergence behaviors. 


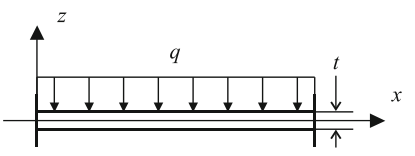

(a)
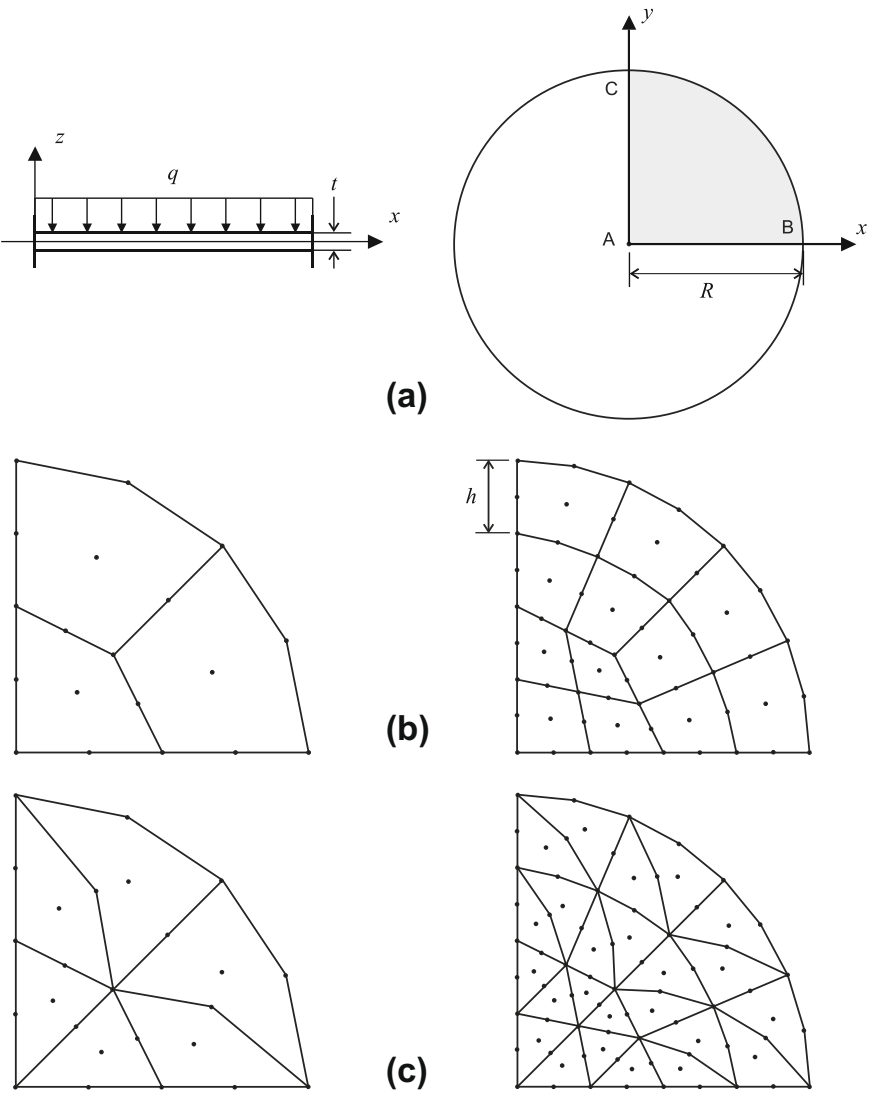

(b)

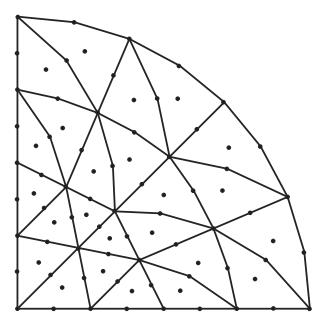

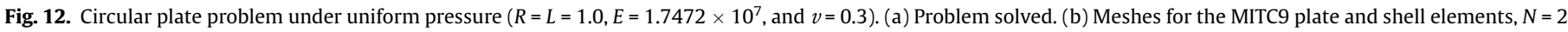

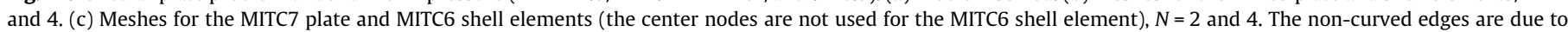
plotting straight lines between element nodes.
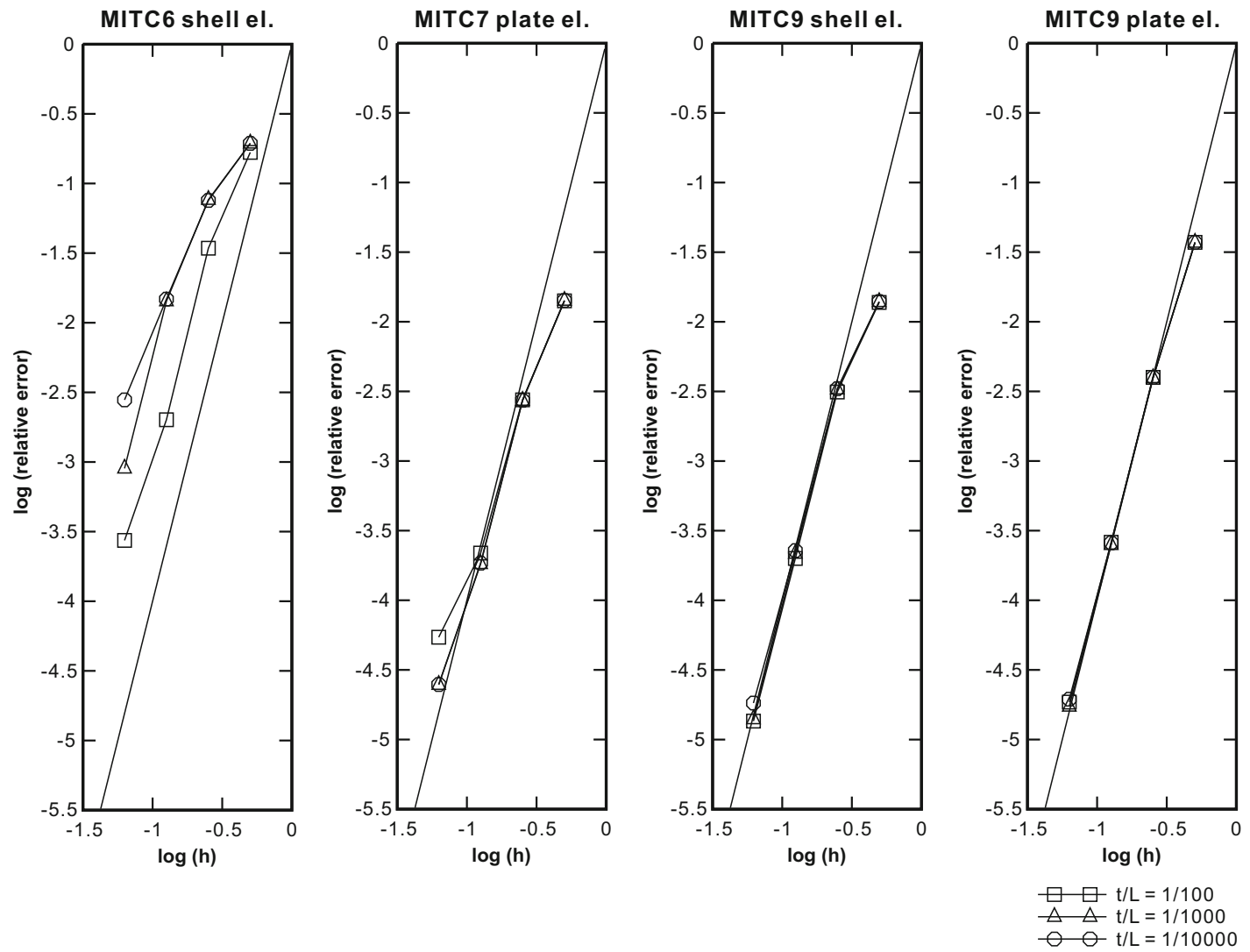

Fig. 13. Convergence curves for the circular plate problem. The s-norm is used. The solid lines represent the theoretical convergence in Eq. (5). 

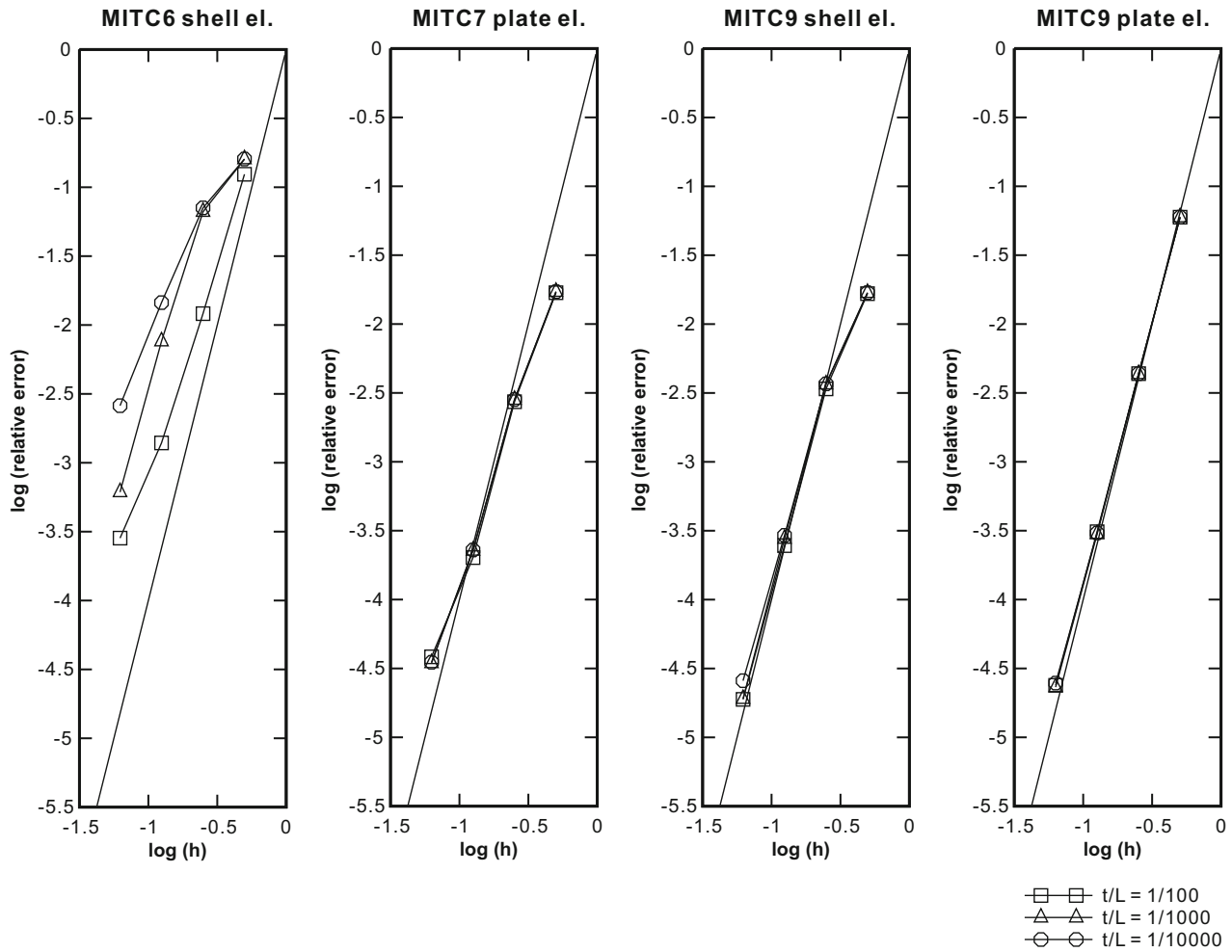

Fig. 14. Convergence of rotations for the circular plate problem. The solid lines represent the theoretical convergence in Eq. (3).
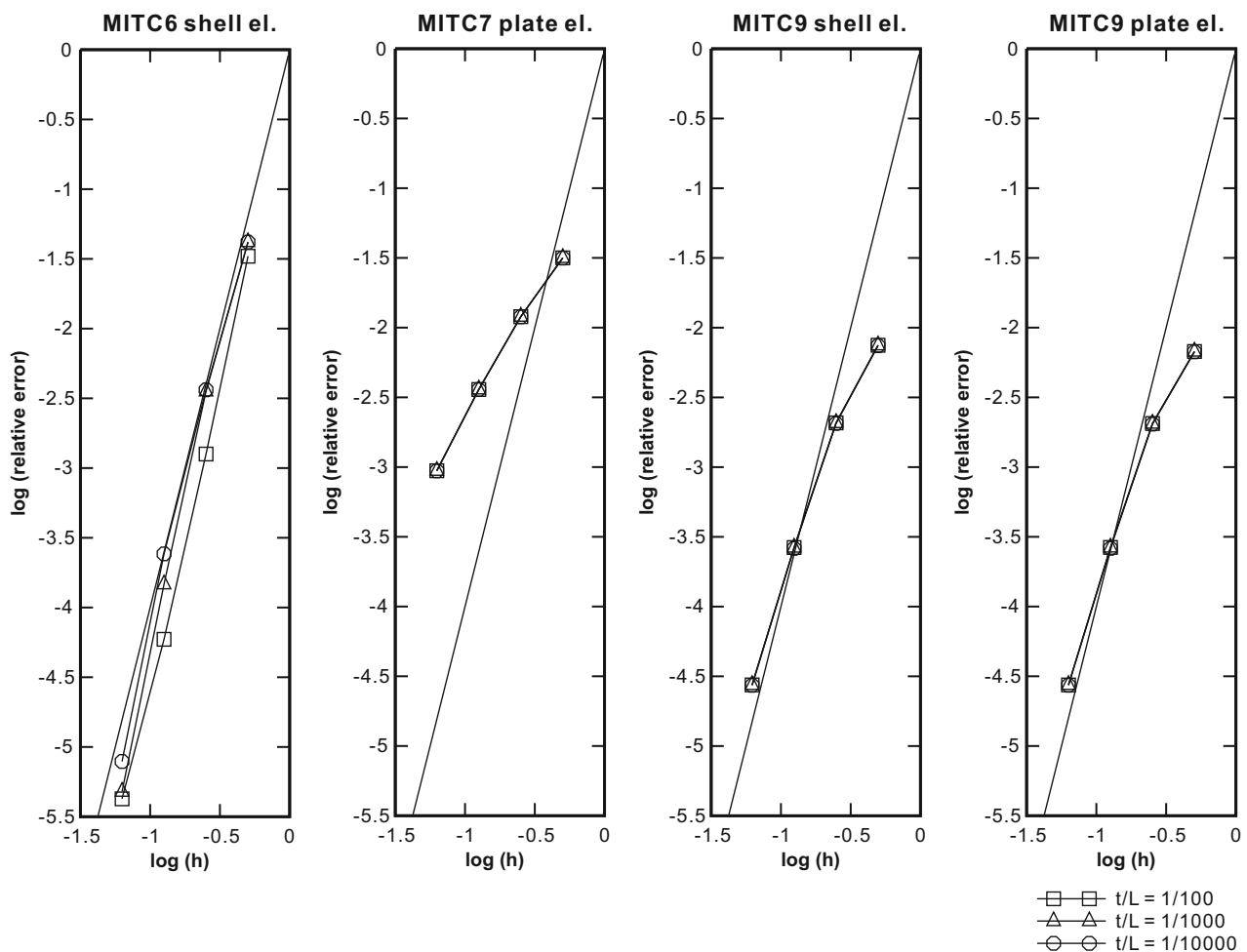

Fig. 15. Convergence of gradient of transverse displacement for the circular plate problem. The solid lines represent the theoretical convergence in Eq. (3).

\section{Numerical study of the MITC plate and MITC shell elements}

In this section, we give the numerical results of three representative plate bending problems using the elements of Figs. 1 and 2. We consider three linear problems of different geometries (square, circular and skew plates). In each case, we use very fine meshes with the MITC9 shell element to establish the reference solutions. Three cases of plate thickness-to-length ratios $(t / L)$ are considered, $t / L=1 / 100,1 / 1000$ and $1 / 10,000$. 

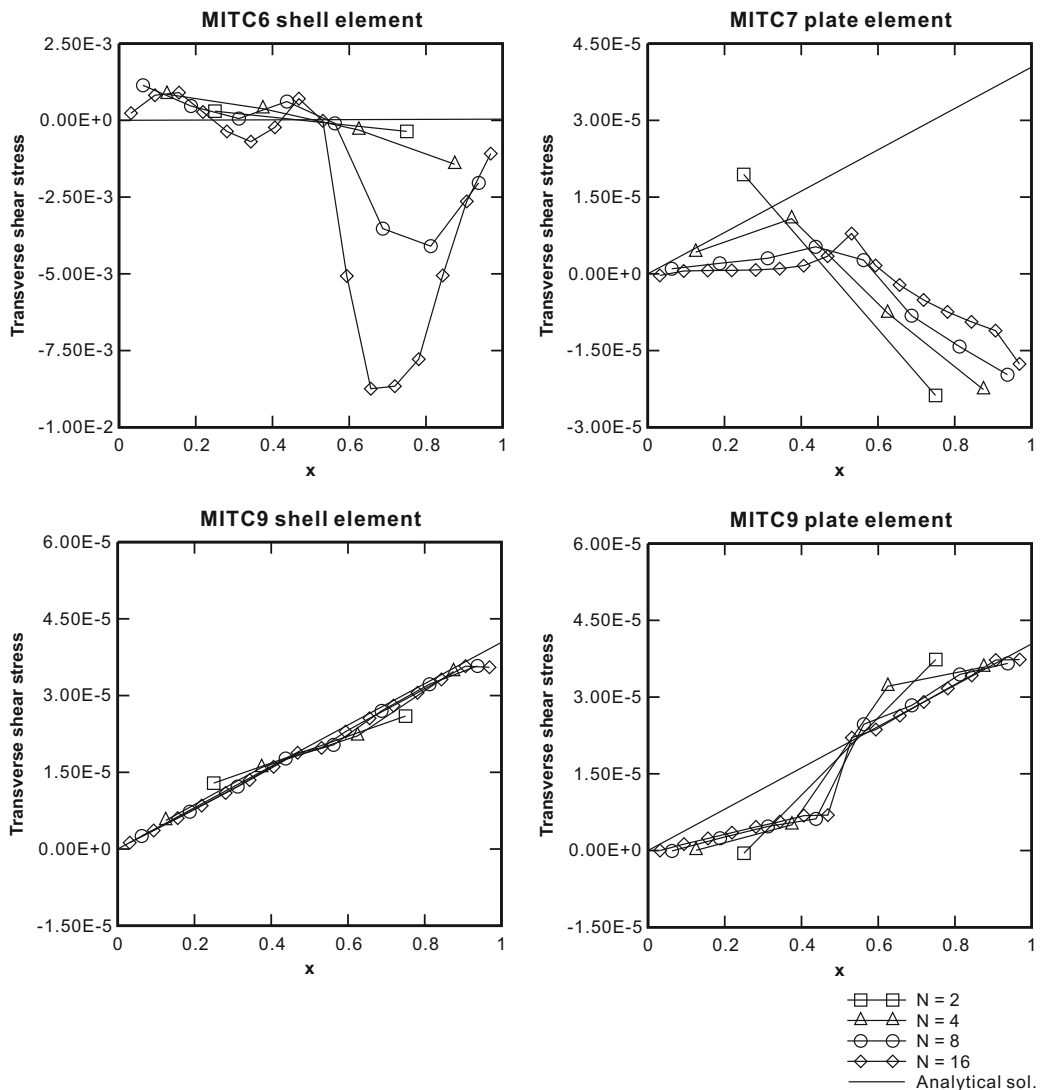

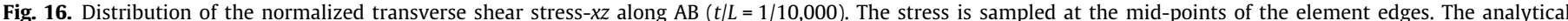
solution is $\tau_{x z}=4.04 \times 10^{-5} x$.
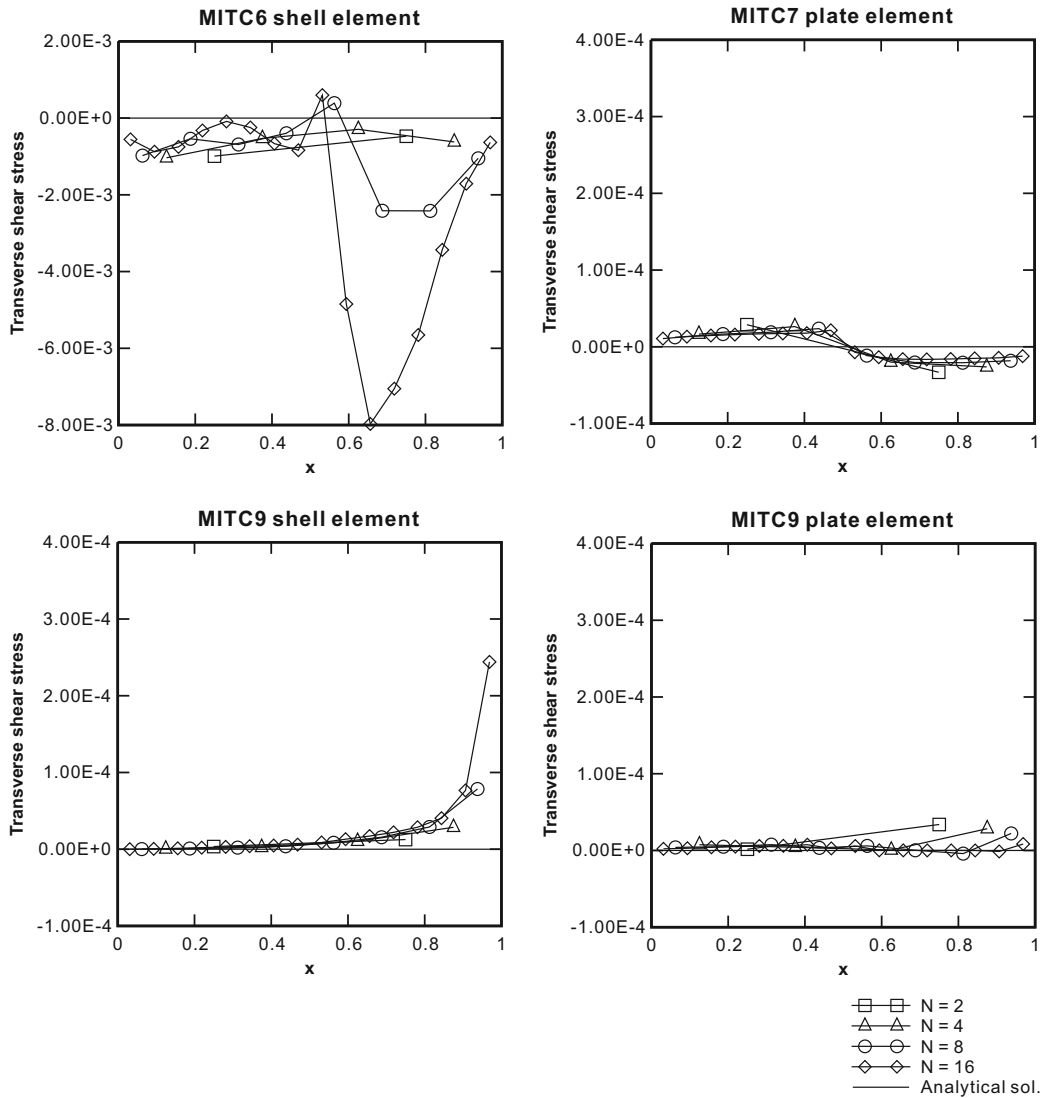

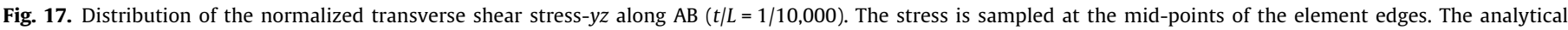
solution is $\tau_{y z}=0$. 


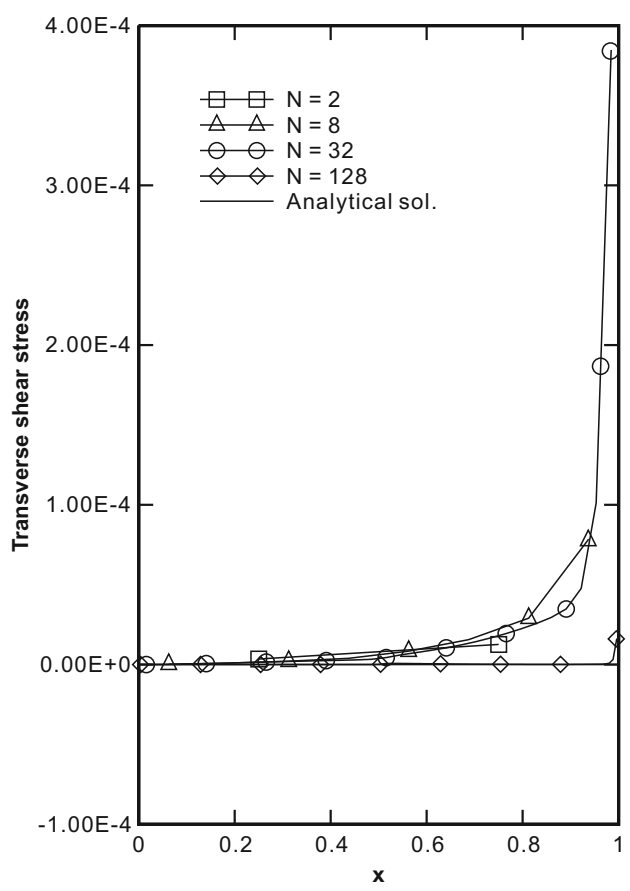

Fig. 18. Convergence of the normalized transverse shear stress-yz in the MITC9 shell element solutions. The analytical solution is $\tau_{y z}=0$.

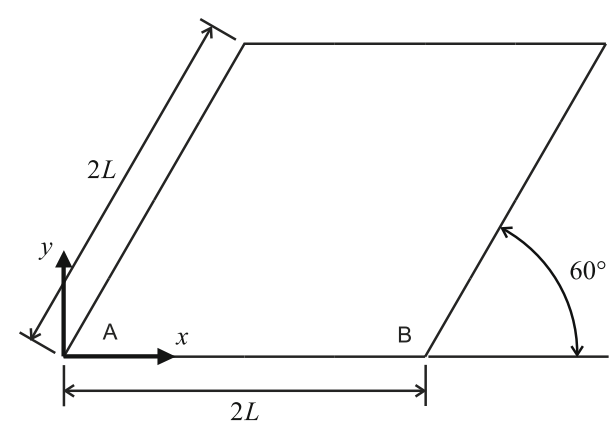

(a)

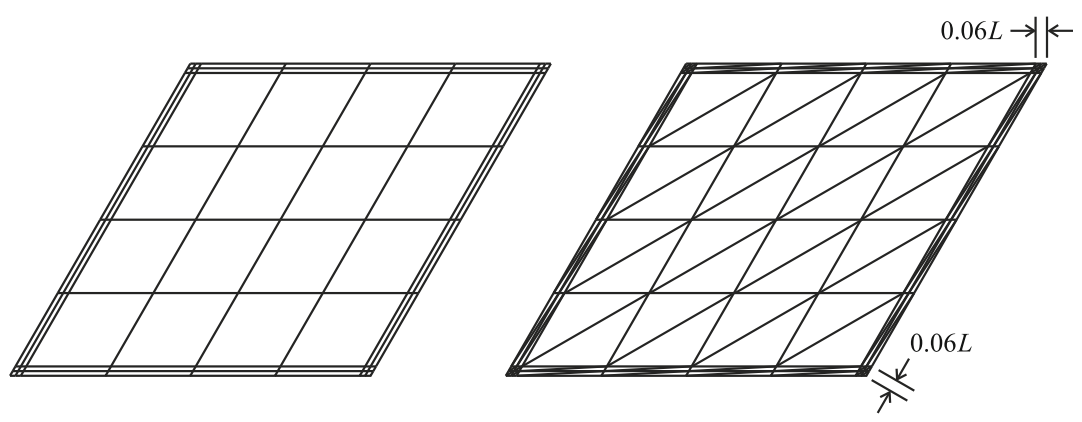

(b)

(c)

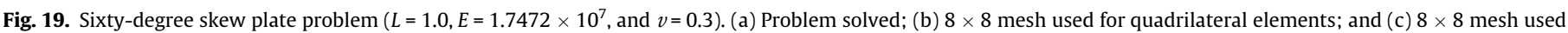
for triangular elements.

\subsection{Analysis of a clamped square plate subjected to uniform pressure}

We consider the plate bending problem shown in Fig. 3. The square plate of dimension $2 L \times 2 L$, with uniform thickness $t$, is subjected to a uniform pressure. All edges are clamped.

Due to symmetry, only a quarter of the plate is modeled (the region $A B C D$ shown in Fig. 3) with the following symmetry and boundary conditions imposed: $\theta_{y}=0$ along BC, $\theta_{x}=0$ along DC, $w=\theta_{x}=0$ along $\mathrm{AB}$ and $w=\theta_{y}=0$ along $\mathrm{AD}$. Note that, along the clamped edges, the soft boundary condition is used and a strong boundary layer does not exist [30]. Fig. 3 also shows the mesh patterns used for $N=4$, the finer meshes are simply obtained by uniformly subdividing the elements. The reference mesh of MITC9 shell elements for the calculation of the norms in Eq. (6) is given by $N=96$.

Figs. 4-6 present the curves of the three convergence measures discussed in the previous section. The MITC9 shell finite element gives uniform optimal (almost ideal) convergence curves for all the cases of thickness $t$ considered.
Figs. 7-11 give the distributions of transverse shear stresses, normalized by the absolute value of the maximum bending stress, for the severe case of $t / L=1 / 10,000$. Of course, these shear stresses are small and difficult to predict accurately. The stresses have been calculated by simply evaluating the shear strains at the points indicated, directly as obtained by the MITC interpolations given in Figs. 1 and 2. Hence no stress smoothing or extrapolation from Gauss points, or other points, has been performed.

Fig. 7 shows the transverse shear stress $\tau_{x z}$ along the edge AB of the square plate calculated at the element nodes. Except for the solutions of the MITC9 shell element, oscillations in the predicted stress are observed. Fig. 8 shows that, when the stress is sampled at the mid-points of the element edges, the MITC9 plate element also gives a reasonable approximation.

Considering all results given in Figs. 7-11, the MITC9 shell element predicts in this problem solution the transverse shear stresses with best accuracy.

The oscillations in the shear stresses shown in Fig. 7 are typical of all subsequent solutions as well, and hence we only show in 
MITC6 shell el.

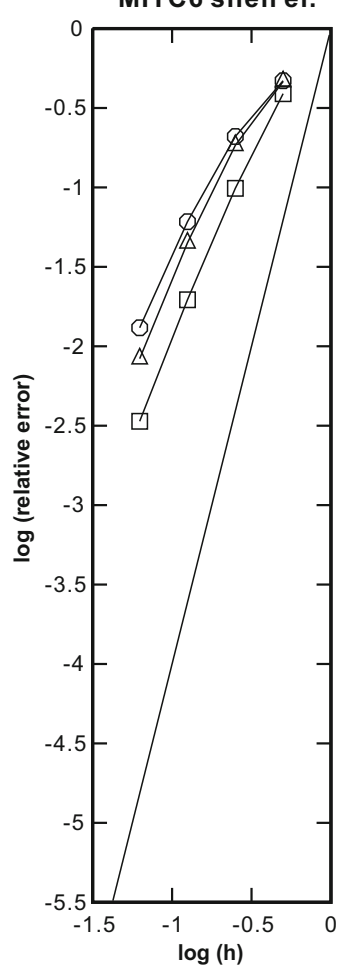

MITC7 plate el.

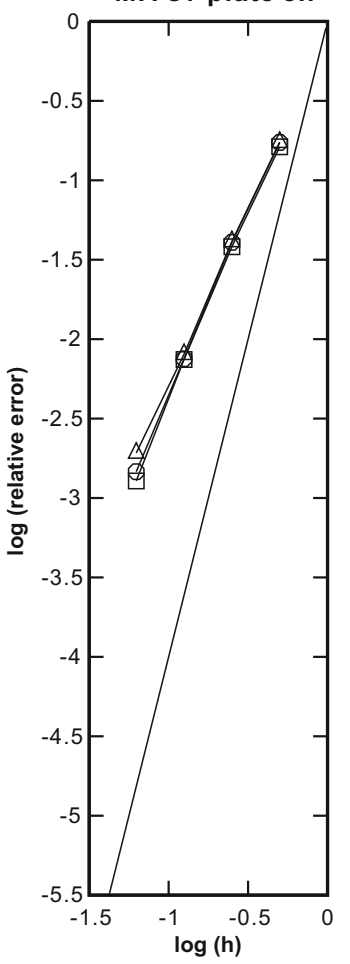

MITC9 shell el.

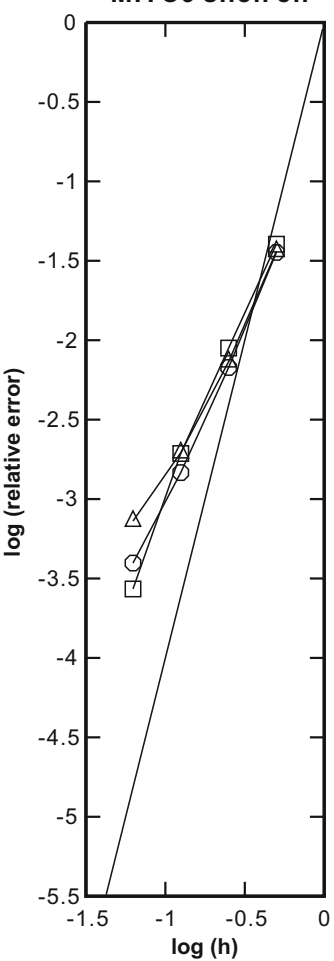

MITC9 plate el.

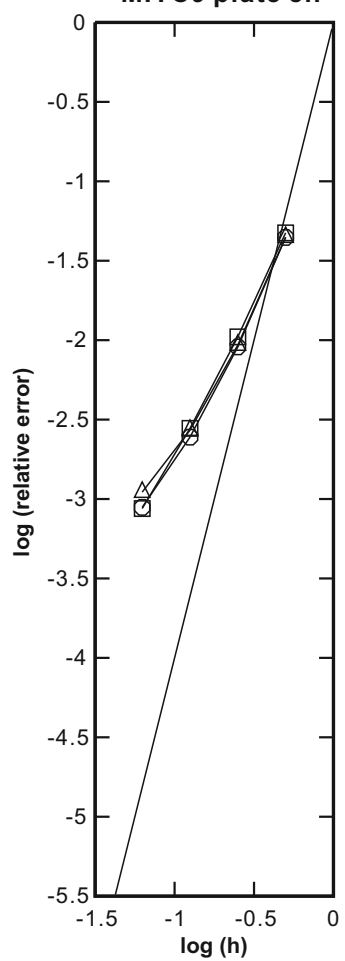

$\square \square \mathrm{t} / \mathrm{L}=1 / 100$

$\triangle \triangle \mathrm{t} / \mathrm{L}=1 / 1000$

$\bigcirc \mathrm{t} / \mathrm{L}=1 / 10000$

Fig. 20. Convergence curves for the $60^{\circ}$ skew plate problem. The s-norm is used. The solid lines represent the theoretical convergence in Eq. (5).
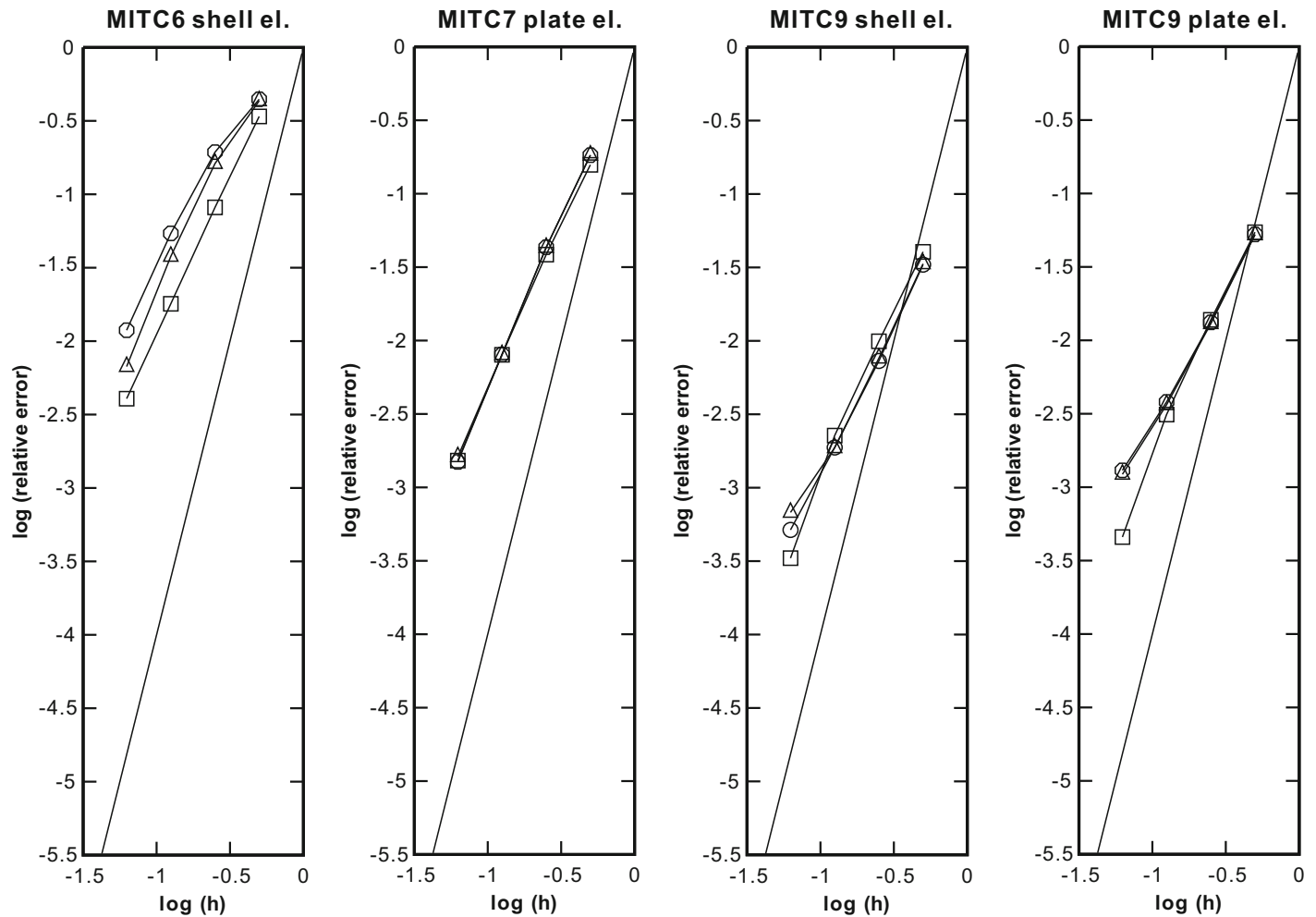

$\square \square \mathrm{t} / \mathrm{L}=1 / 100$
$\triangle \triangle \mathrm{t} / \mathrm{L}=1 / 1000$

$\ominus \mathrm{t} / \mathrm{L}=1 / 10000$

Fig. 21. Convergence of rotations for the skew plate problem. The solid lines represent the theoretical convergence in Eq. (3). 
MITC6 shell el.

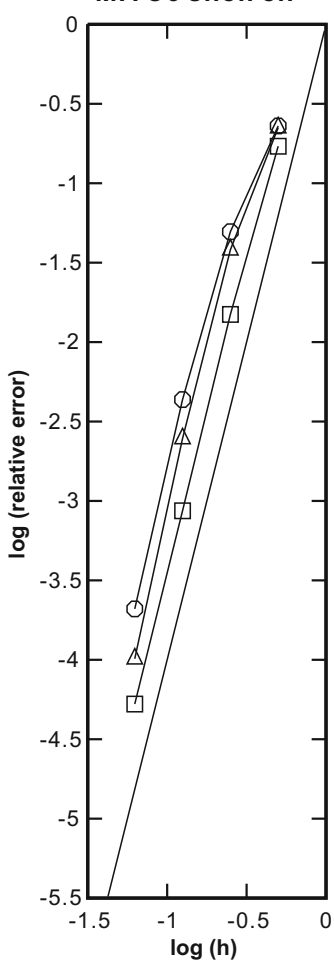

MITC7 plate el.

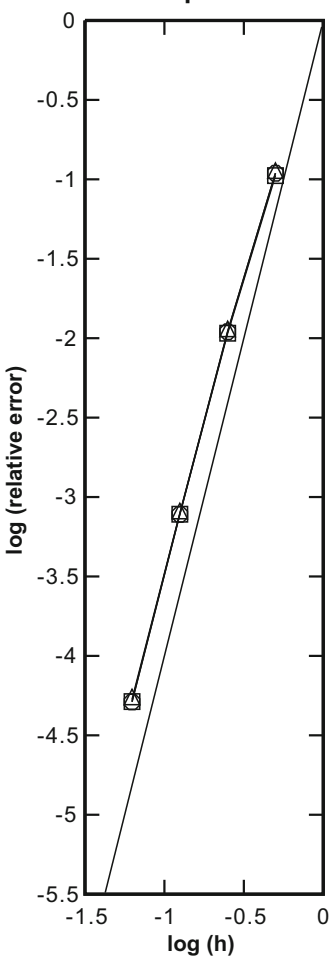

MITC9 shell el.

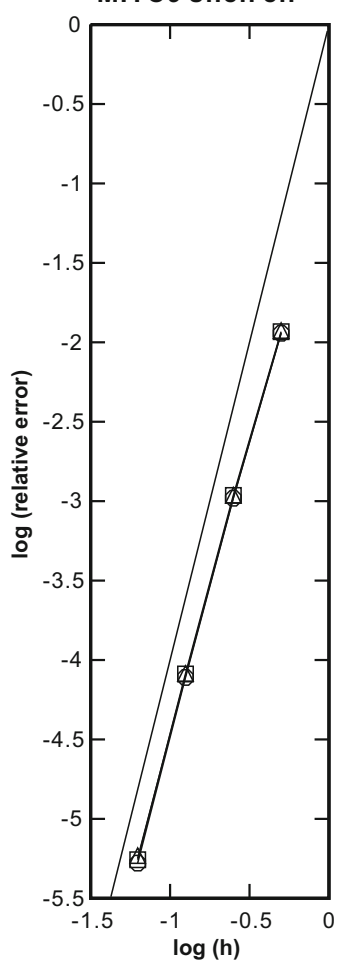

MITC9 plate el.

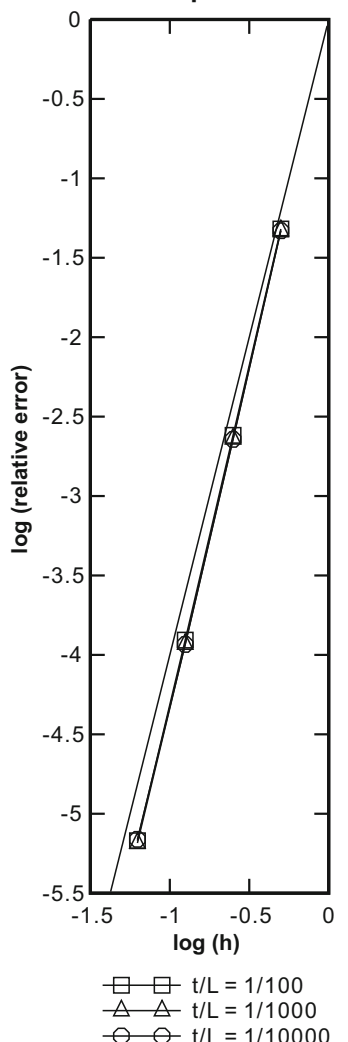

Fig. 22. Convergence of gradient of transverse displacement for the skew plate problem. The solid lines represent the theoretical convergence in Eq. (3).

further results the values calculated at the mid-points of the element edges.

\subsection{Analysis of a simply-supported circular plate subjected to uniform pressure}

We consider a circular plate of thickness $t$ and radius $R$, loaded with a uniform downward pressure, see Fig. 12. The plate is simply-supported along its edge. Using symmetry conditions along $A B$ and $A C$ only one quarter of the plate is modeled (the region $A B C$ shown in Fig. 12). Only $w$ is fixed along $B C$, hence the soft boundary condition is used [30]. Fig. 12 also shows the mesh patterns used for $N=2$ and $N=4$, the finer meshes are simply obtained by uniformly subdividing the elements and representing the circular boundary increasingly more accurately. The reference mesh of MITC9 shell elements used for the calculation of the norms in Eq. (6) is given by $N=96$. But this is a rather simple problem and the analytical solutions for the stresses are available.

Figs. 13-15 present the curves of the three convergence estimates.

Figs. 16 and 17 show the predicted distribution of the transverse shear stresses along the radial direction for the case $t /$ $L=1 / 10,000$. The stresses are evaluated as described in Section 3.1 and are normalized by the magnitude of the maximum bending stress. In this problem, the MITC9 shell element performs well but a rather fine mesh is needed for convergence in Fig. 18. The fine mesh is required to represent the circular boundary with sufficient accuracy. The performance of the MITC9 plate element is acceptable, but for the meshes used the element does not converge well in the prediction of the transverse shear stress $\tau_{x z}$.

\subsection{Analysis of a simply-supported $60^{\circ}$ skew plate subjected to uniform pressure}

Fig. 19 shows the $60^{\circ}$ skew plate we consider. The plate is subjected to a uniform downward pressure. All edges are simply-supported with $w=0$ at the edges, that is, the soft boundary condition is imposed [30].

Of particular difficulty in the solution of this plate problem are the boundary layers and the stress singularity at the obtuse corner B. To approximately resolve the boundary layers we use the graded mesh patterns shown for $N=8$ in Figs. 19b and c. The same boundary layer length meshing is used for all cases $t / L$ considered. The reference solution for the calculation of the norms in Eq. (6) is obtained using the MITC9 shell element with $N=128$.

Figs. 20-22 present the curves of the three convergence estimates. We note that, except for the gradient of the transverse displacement, the optimal rate of convergence is not obtained, which however may be partly due to not resolving the boundary layers well enough.

For the transverse shear stress calculations we consider the plate with $t / L=1 / 100$, since the case $t / L=1 / 10,000$ is too severe for the purpose of this paper. Figs. 23 and 24 show the predicted distributions of the transverse shear stresses along the edge $A B$. The stresses have been calculated as described in Section 3.1 and are normalized by the magnitude of the bending stress $\left(\tau_{y y}\right)$ at the center of the plate. We notice that the stress $\tau_{x z}$ is quite well predicted using the MITC7 plate, and MITC6 and MITC9 shell elements, but the stress $\tau_{y z}$ is more difficult to predict accurately. We show some detailed results using the MITC9 shell element in Figs. 25 and 26.

The solutions in Figs. 25b and 26 are most interesting in that a very steep stress gradient is calculated; the final positive stress 

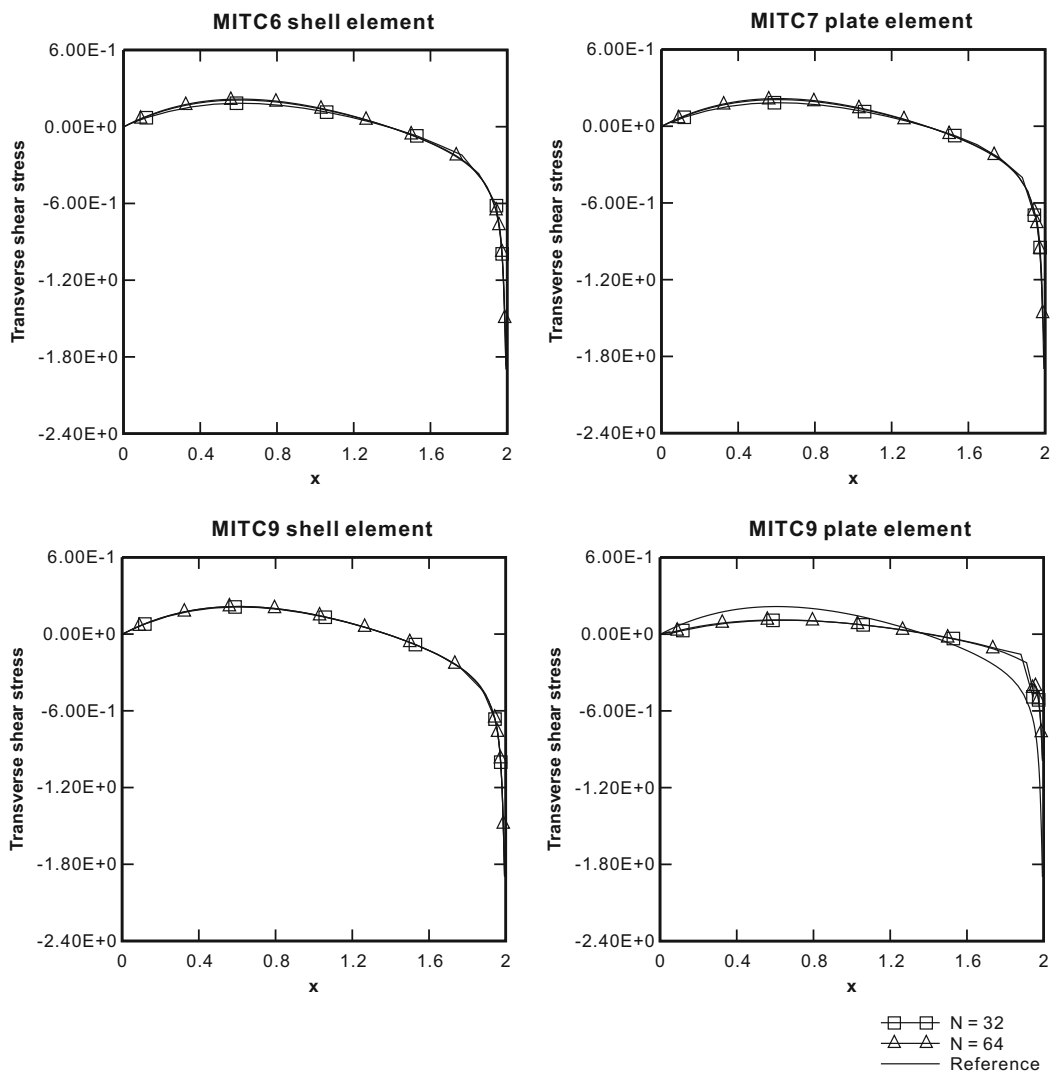

Fig. 23. Distribution of the normalized transverse shear stress- $x z$ along $A B(t / L=1 / 100)$. The stress is sampled at the mid-points of the element edges.
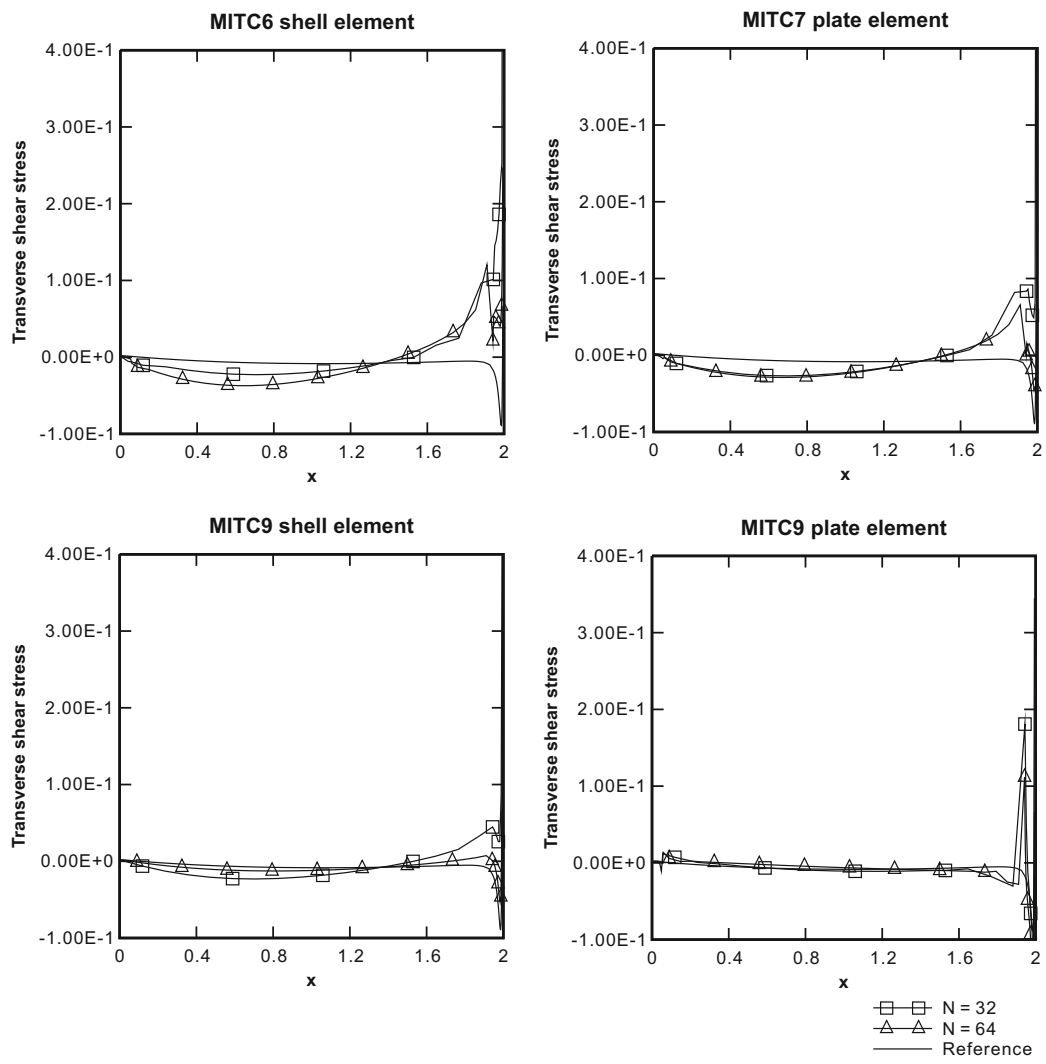

Fig. 24. Distribution of the normalized transverse shear stress- $y z$ along $A B(t / L=1 / 100)$. The stress is sampled at the mid-points of the element edges. 


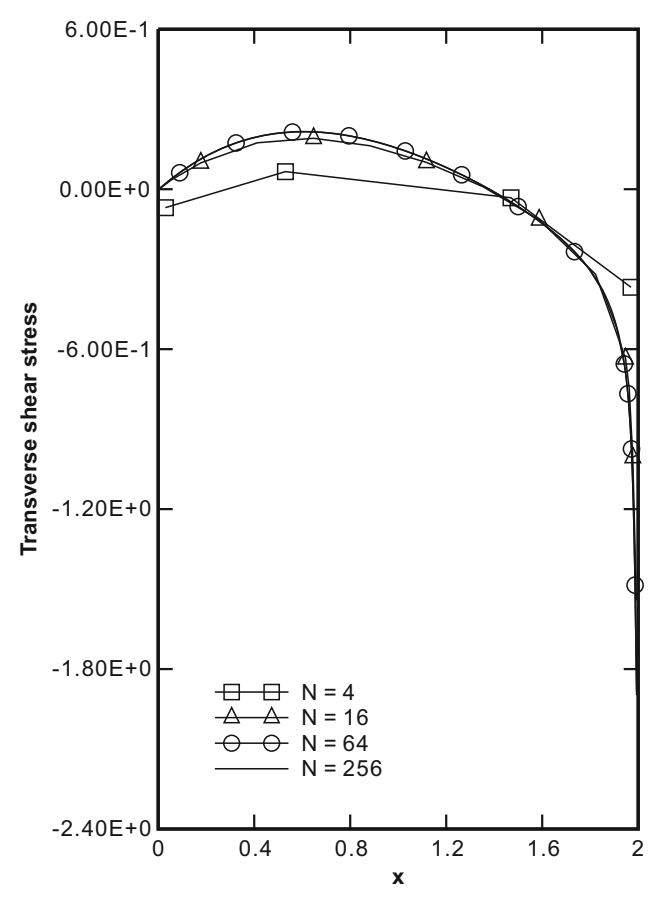

(a)

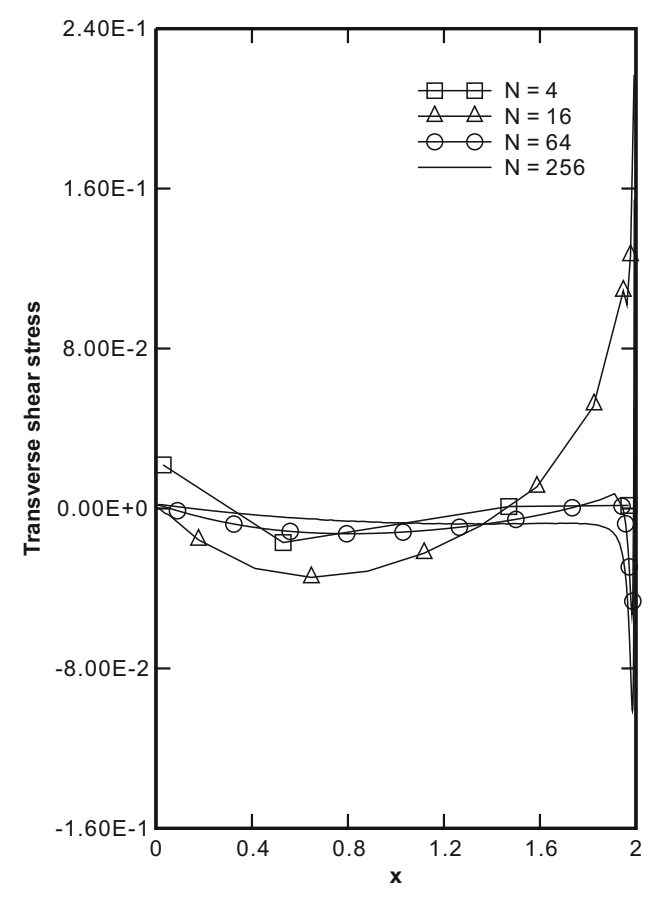

(b)

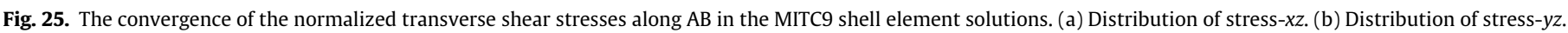
The stresses are sampled at the mid-points of the element edges.

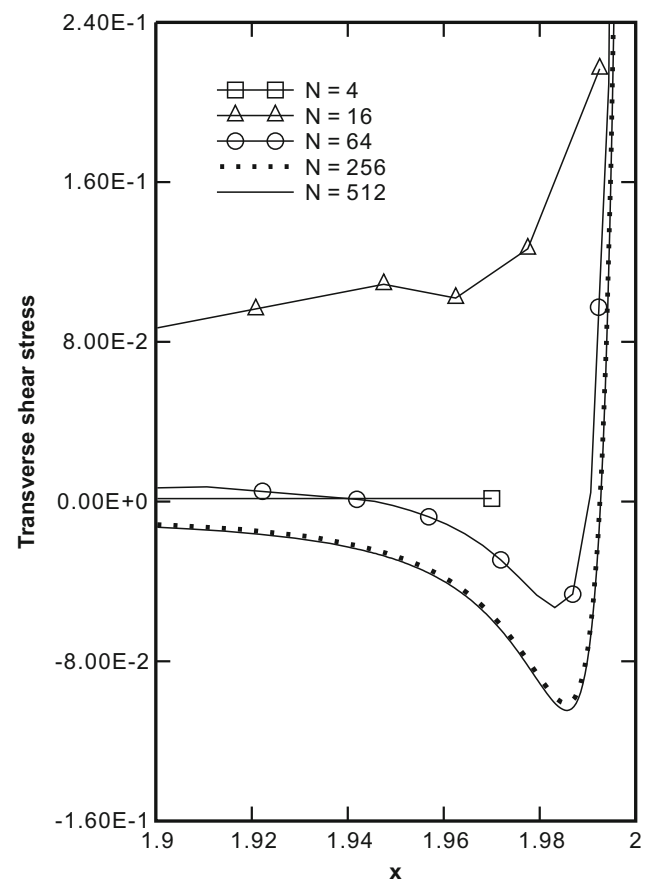

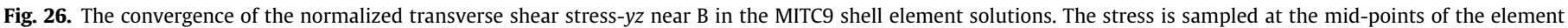
edges.

gradient near B properly obtained when using very fine meshes, see Fig. 26, can hardly be seen in Fig. 25b. Such boundary layers result into the fact that uniform $L^{2}$ convergence of the transverse shear stresses cannot be expected [3].

In Ref. [30], the zero value for the transverse shear stress $\tau_{y z}$ is predicted to occur at the location $x=1.99897$. The results given in Fig. 26 are close to the value.

\section{Conclusions}

The objective in this paper was to compare the performance of the quadratic MITC plate bending and MITC shell elements in some typical linear analysis plate bending test problems. Both element families are based on the Reissner-Mindlin kinematic assumption. While the plate elements have a strong mathematical basis and 
have been proven, and shown in some numerical tests, to be optimal in their performance, irrespective of the plate thickness, the shell elements have largely been formulated based on intuition, some revealing mathematical analysis, and the performance in test problems. The analysis of shells is so complex that general mathematical proofs of convergence of shell finite element discretizations are very difficult to establish.

Since shell elements are generally also used to analyze plates, it is of great interest to compare the performance of the MITC plate and MITC shell elements.

Based on the problem solutions given in this paper, we have found that the MITC7 plate element performs somewhat superior to the MITC6 shell element. This is to be expected since the MITC7 plate element carries additional degrees of freedom. However, overall the shell elements perform as well as the plate elements. Indeed, the MITC9 shell element is performing surprisingly well, and better or equal to the plate elements. The MITC9 shell element convergence rates show virtually optimal convergence, with small constants, and the element also performed well in predicting transverse shear stresses. These stresses are usually orders of magnitude smaller than the bending stresses and are very difficult to predict accurately; indeed, uniform $L^{2}$ shear stress convergence does not exist for the continuous plate problems and can therefore also not be expected for the finite element discretizations.

Using the MITC9 shell element, we also solved for the response of a skew plate and calculated the transverse shear stresses near the obtuse corner where a strong singularity exists. Good convergence was achieved and the solution shows the difficulty to calculate shear stresses accurately.

A valuable endeavor would be to mathematically analyze the quadratic MITC shell elements when used in the linear analysis of plate bending problems. Such an analysis may reveal properties that we have so far not discovered and, indeed, may lead to improved discretization schemes for shell solutions.

\section{References}

[1] Zienkiewicz OC, Taylor RL. The finite element method. Heinemann: Butterworth; 2005.

[2] Bathe KJ. Finite element procedures. New York: Prentice-Hall; 1996

[3] Chapelle D, Bathe KJ. The finite element analysis of shells - fundamentals. Springer; 2003.

[4] Dvorkin E, Bathe KJ. A continuum mechanics based four-node shell element for general nonlinear analysis. Eng Computat 1984;1:77-88.

[5] Bathe KJ, Dvorkin EN. A formulation of general shell elements - the use of mixed interpolation of tensorial components. Int J Numer Methods Eng 1986;22:697-722.
[6] Bucalem ML, Bathe KJ. Higher-order MITC general shell elements. Int J Numer Methods Eng 1993;36:3729-54.

[7] Bathe KJ, Lee PS, Hiller JF. Towards improving the MITC9 shell element. Comp Struct 2003;81:477-89.

[8] Lee PS, Bathe KJ. Development of MITC isotropic triangular shell finite elements. Comp Struct 2004;82:945-62.

[9] Kim DN, Bathe KJ. A triangular six-node shell element. Comp Struc 2009;87:1451-60.

[10] Bucalem ML, Bathe KJ. Finite element analysis of shell structures. Archiv Computat Methods Eng 1997;4:3-61.

[11] Lee PS, Bathe KJ. Insight into finite element shell discretizations by use of the basic shell mathematical model. Comp Struct 2005;83:69-90.

[12] Chapelle D, Bathe KJ. The mathematical shell model underlying general shell elements. Int J Numer Methods Eng 2000;48:289-313.

[13] Bathe KJ, Iosilevich A, Chapelle D. An inf-sup test for shell finite elements. Comp Struct 2000;75:439-56.

[14] Bathe KJ, Iosilevich A, Chapelle D. An evaluation of the MITC shell elements. Comp Struct 2000;75:1-30.

[15] Chapelle D, Bathe KJ. Fundamental considerations for the finite element analysis of shell structures. Comp Struct 1998;66:19-36. 711-2.

[16] Hiller JF, Bathe KJ. Measuring convergence of mixed finite element discretizations: an application to shell structures. Comp Struct 2003;81:639-54.

[17] Lee PS, Noh HC, Bathe KJ. Insight into 3-node triangular shell finite elements: the effects of element isotropy and mesh patterns. Comp Struct 2007;85:404-18.

[18] Pitkäranta J, Leino Y, Ovaskainen O, Piila J. Shell deformation states and the finite element method: a benchmark study of cylindrical shells. Comp Methods Appl Mech Eng 1995;128:81-121.

[19] Havu V, Pitkäranta J. Analysis of a bi-linear finite element for shallow shells. II: consistency error. Math Computat 2003;72:1635-53.

[20] Bathe KJ, Brezzi F. A simplified analysis of two plate bending elements - the MITC4 and MITC9 elements. In: Proceedings, numerical methods in engineering: theory and applications. University College Swansea, Wales; July 1987.

[21] Bathe KJ, Brezzi F, Cho SW. The MITC7 and MITC9 plate bending elements. Comp Struct 1989;32:797-814.

[22] Brezzi F, Bathe KJ, Fortin M. Mixed-interpolated elements for Reissner/Mindlin plates. Int J Numer Methods Eng 1989;28:1787-801.

[23] Bathe KJ, Bucalem ML, Brezzi F. Displacement and stress convergence of our MITC plate bending elements. Eng Computat 1990;7:291-302.

[24] Iosilevich A, Bathe KJ, Brezzi F. On evaluating the inf-sup condition for plate bending elements. Int J Numer Methods Eng 1997;40:3639-63.

[25] Lyly M, Niiranen J, Stenberg R. A refined error analysis of MITC plate elements. Math Models Methods Appl Sci 2006;16:967-77.

[26] Lyly M, Niiranen J, Stenberg R. Superconvergence and postprocessing of MITC plate elements. Comp Methods Appl Mech Eng 2007;196:3110-26.

[27] Bathe KJ. The finite element method. In: Wah, editor. Encyclopedia of computer science and engineering. John Wiley and Sons; 2009. p. 1253-64.

[28] Simo J, Rifai MS. A class of mixed assumed strain methods and the method of incompatible modes. Int J Numer Methods Eng 1990;29:1595-638.

[29] Pantuso D, Bathe KJ. On the stability of mixed finite elements in large strain analysis of incompressible solids. Finite Elem Anal Des 1997;28:83-104.

[30] Häggblad B, Bathe KJ. Specifications of boundary conditions for Reissner Mindlin plate bending finite elements. Int $\mathrm{J}$ Numer Methods Eng 1990;30:981-1011. 